\title{
Étreindre le temps, repriser la parole, déployer l'âme : Le Troisième (2013) et Éternité à coudre (2016)
}

Infléchir la parole peut être un projet ambitieux. Tellermann le fait par exemple en s'inspirant d'autres, en accentuant chaque mot, en développant d'amples séquences traversées par un souffle à la fois vif et tendre, soucieux et attachant, et en inscrivant dans le dire un devenir personnel et collectif, une étreinte des morts et des vivants d'une grande étendue spatiotemporelle que l'on apprend à reconnaître. Le temps mythique du récit ininterrompu nous fait voir des fils qui relient le présent et le passé, l'intime et l'Histoire, les figures d'autrefois et le moi dans l'ici et maintenant. À la relecture, certains de ces aspects narratifs se présentent dès les premiers recueils si dépouillés, au-delà de ce que la quatrième de couverture nomme un journal «poétique » $(T P)$ ou «métaphysique» $(P A)$.

Le Troisième et Éternité à coudre prolongent cette quête dans un cadre un peu à part. Publiés aux Editions Unes, qui privilégient par le format des livres et le grain des papiers la beauté liquide du geste poétique, et qui permettent aux auteurs d'explorer le dire au sein de structures ouvertes et sans bornes, ces deux recueils s'acheminent autrement. Tellermann continue à creuser le chant, mais sous une autre forme. Chaque livre, non paginé, ${ }^{1}$ plonge le lecteur dans une seule séquence, met en évidence la portée de la pensée et la gamme d'émotions et de sensations qui l'animent, sans qu'il y ait besoin de suivre une structure bipartite ou tripartite dont les chants et contrechants laisseraient percevoir une nette progression dans la trame narrative. Un motif peut réapparaître, mais sera autrement nuancé, par exemple celui d'une « éternité à coudre » tantôt édénique et fleurissante, associée à « deux noms » et qui nous aiderait donc à mieux « être » au plan relationnel, tantôt opposée à « l'à pic » brûlant « en nous » qui perturbe et désoriente, qui ne s'efface pas (LT 61, EC 26).

Le Troisième, Éternité à coudre : nos repères sont d'entrée de jeu amoindris. S'agira-t-il de la réunion de contraires ? D'un Je et d'un Tu, d'un ici et d'un

1 Pour faciliter l'analyse, ce chapitre attribuera à chaque ouvrage des numéros de page.

(C) AARON PREVOTS, 2022 | DOI:10.1163/9789004499683_008 
ailleurs relativement perceptibles, mais en même temps à percevoir au travers de symboles et d'abstractions? «Coudre » désignerait quel geste ? La création de rapports avec le temps, la langue, l'Autre ? La redécouverte de silhouettes de seuil en seuil ? Plusieurs caractéristiques importantes relient ces deux recueils et donnent lieu à un titre elliptique. Premièrement, ils abordent la difficulté de faire fleurir la parole en terre fragile. Ils expriment le désir, mettent en avant l'échange et la rencontre, mais ne perdent jamais de vue les écueils du dire, le défi que représente l'objectif d'entrer en dialogue avec l'Autre et ainsi de « coudre à soi le lecteur $»^{2}$ L'absence de bornes narratives accentue ces contraintes, motive le face-à-face plus prégnant avec la progression du récit. Les démarches de Celan dans des circonstances similaires s'avèrent exemplaires, et Tellermann y fait allusion. Ces allusions créent des réseaux sémantiques, deviennent souvent une grille de parole concernant un Autre éloigné, exilé, persécuté, à qui l'on reste malgré tout « encordé » $\left(C E 5^{\circ}\right) .^{3}$ Tellermann reste très proche de Celan en insistant sur « [l]a mort à la frontière de l'intime, la main ouverte sur l'horreur, le nu » en tant que terre symbolique pour l'ensemencement de la parole, ${ }^{4}$ ainsi que sur le côté fin et artisanal de ce travail. ${ }^{5}$

Deuxièmement, ces recueils nous permettent de voir, à travers et au-delà de Celan, le rôle que joue Ossip Mandelstam dans la conception tellermannienne de la parole. L'étroitesse du dire dans ces longues séquences - aux niveaux du lexique, des énoncés, de l'image, des archipels de mots sur la page, de la structure d'ensemble - laisse percevoir en palimpseste des poèmes et des textes poétologiques de Mandelstam. Ceci peut être une évidence, Tellermann en parle d'ailleurs à propos de « ceux qui ont transformé la nuit, l'étoile, la boue et les soleils » (NN 8), mais Le Troisième et Éternité à coudre se prêtent particulièrement bien à un bref détour par lui, dont l'influence sur Celan fut

2 «Esther Tellermann : Le Troisième », <editionsunes.fr>.

3 Cf. $s N$ 47: chercher « d'Est en Ouest / une épaisseur [...] qu'elle soit haleine / corde ou / question ». À propos de cordes réelles ou métaphoriques par lesquelles se lier à l'Autre, celles de la voix qui vibrent et celle, verticale, du souffle, voir aussi Paul Celan, « Yeux d'ardoise » et «Gloire de cendres », Renverse du souffle, op. cit., p. 169 et p. 123. L'adjectif « encordé »se trouve dans le poème « La vérité » du recueil de 1968 Fadensonnen (Soleils-filaments), traduit par Jean Daive dans Strette \& autres poèmes, op. cit., p. 103 : « La vérité, encordée / aux vestiges apparents du rêve ».

4 «Esther Tellermann: Le Troisième», <editionsunes.fr>. Cf. Racine, <editionsunes.fr>, livre d'artiste dont un extrait a été mis en ligne : « Je serrais / les rêves / dans l'une et l'autre / main / afin que s'ouvre / à nouveau / un milieu »; $S N 236$ : guetter une main « qui répand le plus nu / et le revers / de la voix ».

5 Paul Celan, « Lettre à Hans Bender », 8 mai 1960, Le Méridien \& autres proses, op. cit., p. 43-45. 
profonde. ${ }^{6}$ Il ne s'agira pas à son égard d'une étude comparative. Néanmoins, traiter de ce que fait Mandelstam mettra en relief des procédés et enjeux pertinents, surtout concernant le désir de faire sentir la plénitude du cosmos et de la langue avec un strict minimum d'outils. Ce sera une autre manière de souligner chez Tellermann le désir d'embrasser l'univers, d'explorer les étendues du réel et de l'inconscient par le biais d'un langage précis et plein d'arcanes, retenu et lyrique, sobre et cadencé. Par exemple, Mandelstam préférerait que le poème soit dépouillé, épuré, lisible, matériel mais rayonnant, sans « trop de chosification ». ${ }^{7}$ Comme Celan après lui, il évite tout maniérisme, privilégiant un phrasé restreint, des situations élémentaires, des mots-cristaux qui exigent une réflexion constante de la part du lecteur et des vers où le «moule sonore de la forme $»^{8}$ rendra sensibles, vibratoires, le peu d'images qu'il recèle. Ce sont des poèmes où « parler, c'est être en route » vers quelqu'un d'inconnu qui écoutera ${ }^{9}$ : on est guidé par un locuteur souhaitant nous rendre attentifs au dialogisme, à des circonstances relativement concrètes, avec des mots qui «err[ent] librement $»^{10}$ et «nous éveill[ent]» constamment, comme «des tiges » sortant d'un faisceau. ${ }^{11} \mathrm{Si}$ s'accorder au temps reste une préoccupation,

6 Voir par exemple les traductions qu'a faites Celan des poèmes de Mandelstam, dans Paul Celan, Gesammelte Werke, t. V, hrsg. von Beda Allemann und Stefan Reichert unter Mitwirkung von Rolf Bücher, Frankfurt am Main : Suhrkamp, 200o, p. 47-161. Il convient d'ailleurs de noter l'influence de Mandelstam sur l'œuvre celanienne dans son ensemble. Cf. Bernhard Böschenstein, «Le Méridien et ses matériaux : le centre de la poétique de Celan », Paul Celan, Europe 1049-105o (sept.-oct. 2016), p. 29-37 (34) : «Le Méridien est plein d'allusions qui expriment les changements qui dépendent des formes de communication. Nous constatons ici l'influence de Mandelstam et de sa conception du poème comme une bouteille jetée à la mer, le mouvement vers un être inconnu étant dès lors le moteur de la poésie. » Celan a traduit de nombreux poètes de langue française : Apollinaire, Artaud, Baudelaire, Breton, Cayrol, Césaire, Char, Daive, Desnos, Du Bouchet, Dupin, Éluard, Maeterlinck, Mallarmé, Michaux, Nerval, Pastoureau, Péret, Rimbaud, Supervielle, Valéry (Gesammelte Werke, t. IV).

7 Ossip Mandelstam, «Le mot et la culture », De la poésie, trad. et postface Christian Mouze, «Mots pour De la poésie » d'Olivier Gallon, Paris : La Barque, 2013, p. 7-14 (12): « N'exigez pas de la poésie trop de chosification, de concrétude, de matérialité. [...] Le mot vivant ne désigne pas l'objet, mais choisit librement comme pour y habiter telle ou telle signification concrète, telle matérialité, tel corps bien-aimé. »

Ibid.

9 Ossip Mandelstam, « De l'interlocuteur », De la poésie, op. cit., p. 21-30 (29) : « [L]a poésie dans son ensemble se dirige toujours vers un destinataire inconnu plus ou moins éloigné, dont le poète ne peut mettre en doute l'existence sans douter de lui-même. »

10 Ossip Mandelstam, « Le mot et la culture », De la poésie, op. cit., p. 12.

11 Osip Mandelstam, Voronezh Notebooks, trans. and intro. Andrew Davis, New York : NYRB, 2016, p. xvi, extraits de « Conversation sur Dante ». Nous traduisons de l'anglais. 
c'est à cause d'une conscience aiguë que la parole est passée par des expériences destructrices. ${ }^{12}$

Troisièmement, Tellermann met en avant un point de vue sur le temps qui s'inspire en partie de Nerval. Dans l'univers nervalien, s'accorder au temps peut vouloir dire reconnaître l'à-venir du temps, le jaillissement spatiotemporel qui peut s'inscrire dans la relation à soi, au cosmos, à l'éternel, aux morts et aux vivants. Chaque mot-symbole du poème doit communiquer le caractère mystérieux et puissant de cette relation, sceller dans le dire un bouquet d'idées et de sentiments venant tant de l'inconscient que des domaines du mythe ou de la religion. ${ }^{13}$ Ainsi en est-il pour Le Troisième comme titre, vraisemblablement inspiré par le premier vers d'« Artémis » de Nerval : «La Treizième revient... C'est encor la première. ${ }^{14}$ Nerval y met en scène une Artémis multiple : Artémis divinité de la nature sauvage, de la chasse et de la lune ; Artémis-Hécate divinité de la magie, des enchantements et du royaume des morts, «sainte de l'abîme ». Elle incarne la bien-aimée qui le guidera dans le noir et le doute, lui fera dépasser dans le songe toute limite temporelle, par exemple le passage entre le douze et le un, le jour et la nuit, ce monde-ci et l'autre monde. Nous assistons au retour cyclique d'une « Treizième » inconnue, énigmatique, éternelle, liée dans le Tarot à l'arcane XIII, celui de la Mort, que ne porte pas de nom. Il y aurait des liens significatifs à faire avec Aurélia ou Le Rêve et la Vie, où la lune se fait le refuge « de toutes les âmes sœurs » de celle du narrateur, astre « peuplé d'ombres plaintives destinées à renaître un jour sur la terre... ». ${ }^{15} \mathrm{En}$

12 Cf. Bernhard Böschenstein, «Le Méridien et ses matériaux : le centre de la poétique de Celan », op. cit., p. 35, où Böschenstein commente ces aspects de la poétique celanienne vis-à-vis du contexte historico-littéraire allemand. Il convient de souligner également le cas de Mandelstam, qui a souffert à cause de l'antisémitisme - mais, d'un autre côté, n’a pas su ménager autrui - et a connu l'exil vers la fin de sa vie.

Cf. John W. Kneller, « The Poet and His Moira : "El Desdichado" », PMLA 75.4 (Sep. 1960), p. 402-09 (402). Kneller parle de «word-symbols» (mots-symboles) et du «cluster of ideas and feelings » (bouquet d'idées et de sentiments) qui émane de presque chacun d'entre eux.

14 Gérard de Nerval, «Artémis », Les Chimères, in Jean Richer, Gérard de Nerval, op. cit., p. 100-11 (106). Nos remarques ici sur Nerval s'appuient, dans l'ordre, sur les sources suivantes : J. W. Kneller, «"Artémis" », The Poem Itself: 150 of the Finest Modern Poets in the Original Languages, ed. and intro. Stanley Burnshaw, with Dudley Fitts, Henri Peyre and John Frederick Nims, Little Rock: U of Arkansas P, 1995, p. 6-7 ; Tina Malet, « "Artémis" de Gérard de Nerval : une grille de lecture», <etudes-litteraires.com>; Lucette Finas, « Une lecture d'Artémis" de Gérard de Nerval », Le Cahier (Collège international de philosophie) 6 (oct. 1988), p. 85-102 ; et Rosanna Warren, « The "Last Madness" of Gérard de Nerval», The Georgia Review 37.1 (Spring 1983), p. 131-38.

15 Gérard de Nerval, Aurélia ou Le Rêve et la Vie, Seconde Partie, VI, in Les Filles du feu suivi de Aurélia, préface de Béatrice Didier, Paris : Flammarion, 1997, p. 344. 
outre, le poème «Artémis » reflète les crises de santé de Nerval. L'effacement des frontières du temps, loin de figurer un simple trope littéraire ou un idéal romantique, y correspond aussi à la transcription d'une descente aux enfers personnelle, psychique, existentielle, d'un face-à-face avec des troubles psychiques qui lui coûteront la vie. Il se comprend au travers de doubles de luimême, d'êtres multiples dont les flottements identitaires font écho aux siens.

Tellermann indique son intérêt pour « Artémis » en le lisant lors d'un entretien avec Sophie Nauleau sur France Culture. ${ }^{16}$ Les parallèles entre les problématiques que nous venons d'évoquer et les recueils Le Troisième et Éternité à coudre sont multiples. Les enjeux sont profonds, en particulier celui de Celan en tant que double imaginaire de Tellermann : celui qui s'identifie aux trépassés, fait de son mieux pour leur parler, sert de passeur de mémoire pour que leur parole nous appartienne et vice versa, s'y prend dans « une langue sublunaire, gris-ciel et gris-cœur, traversée par le souffle dans le temps $\gg .{ }^{17}$ À la différence de Nerval, le dire chez Celan et Tellermann est relativement terrestre, dépouillé de ces larges métaphores qui dépasseraient symboliquement le réel. Néanmoins, le temps y est assez souple, lié à ce qui surgit du langage ou de l'inconscient, accordé non pas à l'horloge mais aux fils dialogiques qui vont se tisser entre soi et l'Autre. Comme l'explique Celan au sujet de Mandelstam, la voix est « sonore et sourde à la fois », le lieu du poème «ici, ici-bas, dans le temps », son orientation celle d'un espace de dialogue où l'interpellé « devient présent, se rassemble autour du moi qui l'interpelle et le nomme $» .^{18}$ Dans Le Troisième, Tellermann se tourne souvent vers Celan - double jamais nommé dans l'espoir de voir « fleuri[r] les / métamorphoses » (LT $\left.5^{2}\right)$. Grimper «plus bas / dans la glaise / coudre / morceau de symbole / et d'étreinte » $\left(L T 5^{2}\right),{ }^{19}$ c'est tenter d'effectuer des recommencements terrestres, poursuivre un peu comme Nerval un dialogue avec l'Autre en soi, relation que symbolise le chiffre trois dans l'image éponyme du «Troisième ». Cette image recèle des gestes

16 Sophie Nauleau, « Entretien avec Esther Tellermann », France Culture, émission « Ça rime à quoi ? », entretien et lectures de poèmes autour du livre Le Troisième, <franceculture.fr >, 23 mars 2014.

17 Paul Celan dans Bernhard Böschenstein, «Le Méridien et ses matériaux : le centre de la poétique de Celan », op. cit., p. 35 .

18 Paul Celan, « La poésie d'Ossip Mandelstam », op. cit., p. 10.

19 Il convient de signaler la dimension poétologique de ces vers. Grimper « plus bas », là où traîne un «morceau de symbole / et d'étreinte » $\left(L T 5^{2}\right)$, c'est aussi, suivant une logique celanienne, «grimper / dans le sans-images », s'appuyer sur le souffle - sur l'énergie, le mouvement et la fragilité de celui-ci - en assumant le néant, en visant l'essentiel dans ses prises de parole et en résistant aux courants poétiques moins profonds, pleins d'artifice, trop prompts à filer des métaphores ou à donner des leçons. Voir Paul Celan, «Du fond des marais », Partie de neige, op. cit., p. 109 et p. 223-25. 
sacrés : se soucier des morts, les veiller, les étreindre au sein d'un temps proche de la légende, planter « des fleurs de cailloux sur les tombes ${ }^{20}$ prononcer une bénédiction aux accents familiaux ou générationnels tel Celan dans « Devant une bougie » : « au nom des trois dont les anneaux / [lui] brillent au doigt [...] au nom du troisième qui met des pierres / blanches en tas ».21 Si Le Troisième évoque une crise, la difficulté d'atteindre « ceux d'en bas » (LT 99), Éternité à coudre nous rapprochera un tant soit plus « de la permanence du monde dans les cendres », ${ }^{22}$ en accentuant un retour à cette relation avec Celan comme âme sœur et bien-aimé et, de même, avec la Poésie comme espace où nos solitudes peuvent se partager à travers le temps.

Quatrièmement, la notion de « coudre » joue un rôle fondamental dans ces deux recueils. De nombreux indices y convoquent l'urgence du dire, ainsi que la tendresse et la fragilité qui caractérisent les pas faits vers l'Autre. Sans qu'il s'agisse d'une métaphore filée, l'idée de coudre ne cesse de résonner, tant dans les poèmes que dans les notices sur le site web de l'éditeur. La première notice nous fait penser à l'Être brisé, à un face-à-face avec l'Histoire et avec le langage à l'égard de « [1] mort à la frontière de l'intime $[\ldots]$ l'horreur, le nu », ${ }^{23}$ peutêtre même au tableau expressionniste Le Cri (1893) d'Edvard Munch (18631944) en plus violent encore : « [l] e cri par dessus le bâillon ». ${ }^{24}$ On comprend bien qu'il y aura un discours sur l'amour, mais qui s'infléchira cependant vers des propos sur l'indicible, sur des gestes parfois désespérés pour recoudre la «plaie» de la mort comme «blessure ». ${ }^{25}$ Comme chez Nerval et Celan, il sera question de s'appuyer sur l'Autre pour combattre la folie, pour résister à la perte de tout sens : le porter à soi pour repriser nos liens au cosmos et à la parole, déployer son « murmure » dans le ciel, «à hauteur des cercles », là où les astres se meuvent vers des méridiens. ${ }^{26}$ La deuxième notice suggère qu'il y a

20 «Esther Tellermann : Le Troisième », <editionsunes.fr>. Cf. LT 71 quant au renouvellement souhaité de tels « gestes ».

21 Paul Celan, [De seuil en seuil], Choix de poèmes, op. cit., p. 97-99 (99).

22 «Esther Tellermann : Éternité à coudre», <editionsunes.fr>.

23 « Esther Tellermann : Le Troisième », <editionsunes.fr >.

24 Ibid.

25 Ibid.

26 Ibid. Nous commentons des vers qui accompagnent le texte publicitaire : «Je voulais te porter / jusqu’à moi / décupler / les odeurs / et les pôles / voulais déployer / ton murmure / / à hauteur des cercles » (LT 97). Cf. Paul Celan, « En l'air là-haut » (le dernier poème du recueil La Rose de Personne), Choix de poèmes, op. cit., p. 220-23, et p. 357n8 : « Sternzeit, le temps stellaire, connote [...] l'étoile juive et l'époque de la Destruction : le temps qu'il faut pour que le centre d'un astre repasse au méridien de la sphère céleste. » Il se peut que Tellermann pense par exemple à l'« Ici » d'où se ferait entendre le murmure des « divisés avec leurs / bouches éblouies », des « dispersés », des « étrangers à vie», des « errants 
crise - d'identité, de société, de vers. En lisant les propos sur la tâche orphique de l'auteur, nous pensons tant à Voyageur contemplant une mer de nuages (1818) du peintre Caspar David Friedrich (1774-1840), où le corps entier d'un sujet «[d]ebout sur la rive» métaphorique semble méditer «nos éternités et nos solitudes », qu'à l'impossible de la Shoah et l'obligation d'expier des fautes par une « parole rituelle ». ${ }^{27}$ La tâche orphique sera également beckettienne : «On emplit l'éternité dans des sacs, dans les sacs pleins de l'histoire. Trop lourds à porter. Qui pèsent sur nous et nous essayons malgré tout d'arracher quelque chose du monde ou du langage. $»^{28}$ On se trouve à mi-chemin entre le sacré et l'absurde, le rite et le geste courageux mais vain, un peu fou. ${ }^{29}$ En même temps, quelque chose de charnel et d'intersubjectif nous rassure. Le partage prévaudra, une relation se renouera, dans la mesure où, métaphoriquement, la parole de l'Autre se fera «abri », pain partagé, réponse aux impasses, lieu des « noms mâchés », ${ }^{30}$ chemin vers l'aperception de seuils et donc vers le calme retrouvé. La notion de « coudre » fait écho en somme à l'acte poétique en tant que rite simple ou solennel, bienfaisant mais parfois difficile à mener à bien. Ce vocable est souple. Dans Le Troisième et Éternité à coudre, ses dimensions spatiotemporelles, intersubjectives, spirituelles et poétologiques seront particulièrement pertinentes.

L'un des sens intersubjectifs du mot mérite des remarques sur l'actualité littéraire et socioculturelle. Dans ces deux recueils, il s'agit en quelque sorte d'une relation épistolaire destinée à faire apparaître ce «Elle» $(E C$ 8, 28, 29, 36), la Poésie qui témoigne, et qui persévère dans ce témoignage malgré le

conduits à travers le désert stellaire Âme » (223). Coudre à soi l'Autre, ce serait ainsi repriser en même temps le fil de la parole qui était autrefois le leur, l'épisser au nôtre, accueillir l'étrangeté, l'inhabituel.

27 « Esther Tellermann : Éternité à coudre », <editionsunes.fr>.

28 Ibid.

29 Ibid.

30 Ibid. La notion de «mâcher » des noms renvoie à celle de la couture. Mâcher, c'est créer à travers l'écriture : à partir de la bouche, de la langue, des lèvres, des sens, du souffle, de tout le système nerveux et respiratoire, ainsi que de l'œil qui voit, des doigts et des mains qui tiennent le stylo et pèsent chaque mot comme pierre à poser, à offrir. Résultat : le drapeau de mots qui tient le chant, le nourrit, l'unit, le fait perdurer. Dans les recueils euxmêmes, il s'agit d'un Tu ou d'un Vous qui est « mâché » : « Qui fûtes-vous [...] c'est vrai tu fus / ma salive / étais-tu ce qui ne / fut / moi / orgues où puisent / les aveugles » (LT 38); « vous avais-je / arraché / à l'ordre du fou / vous avais-je / mâché / avec l'écriture » (LT 51); « je vous mâchais / avec l'écriture » $(E C 17,32 ; \mathrm{cf} .16,30,33)$; «je voulais / vous mâcher avec / l'écriture » (EC 33). Cf. Paul Celan, «Parler avec les impasses », Partie de neige, op. cit., p. 55, et «Avec leurs mâts chantés vers la terre », Renverse du souffle, op. cit., p. 27 . Quant à l'« abri » mentionné dans la notice, cf. EC 40, « Mots furent notre / auge notre / abri ». 
temps qui passe. Sous l'énigme, voilà une finalité charnière. Poser des cailloux, c'est signaler par la bouche et le corps les relations qui nous font exister, raconter par les signes l'affect au-dedans et au-dehors de l'étant. ${ }^{31}$ C'est faire un pas vers l'Autre dans sa ressemblance ainsi que sa différence, selon l'aimantation qui fait de nous des êtres sociaux et tisse nos identités multiples. Le Troisième et Éternité à coudre mettent en valeur l'intersubjectivité de façon à faire de celle-ci le moteur de la poésie. ${ }^{32}$ Le premier recueil regrette les ponts relationnels momentanément coupés; l'autre volet du diptyque voit l'auteur à même de les reconstruire. Dans les deux cas, les vocables et énoncés empruntés à Celan rendent le dire sinon épistolaire au sens strict, du moins hautement dialogique.

Il est également important de mettre en évidence ce qu'apporte cette poétique à la poésie contemporaine. Comme nous avons pu le constater, le langage peut incarner l'Autre proche et lointain qui nous relie. Les auteurs de diverses traditions font valoir tantôt l'impossibilité de faire un avec la langue, tantôt les joies de garder le contact avec sa matière et ses ressources. Les livres de Tellermann nous rappellent la pertinence de ces diverses approches, mais il est possible que l'accent mis parfois sur une relation épistolaire - un mouvement vers l'Autre comme être bien-aimé mais inconnu - les situent à part dans cet horizon poétique. Or, l'intérêt croissant pour le bien-être de la planète les rend on ne peut plus nécessaires. Nous assurer une relation avec autrui, à l'égard de la langue et des aspects très humains du poème, s'accorde parfaitement avec l'esprit du temps écologique. Veiller sur la parole et sur les disparus en tant qu'ils font partie intégrante de celle-ci, correspond à l'éthos de notre ère qui consiste à aider la planète comme s'il s'agissait de Gaïa, de la terre mère, d'un Autre «énigmatique-maternel» mais fondateur qui nous réveille. ${ }^{33}$ Enfin, c'est également dans ce sens que l'on peut considérer l'angoisse qui fait surface

$31 \quad$ Par exemple : « devant la porte ceux / qui revinrent des exils / nous les raconterons / dans les cailloux / et la glaise [...] nous serons leurs / messagers dessus / le vivant » (LT 74); «Puis un / désir / d'eau / et d'âme / de cailloux blancs / toi / sœur / te fais bruire / tant / fus / ma lumière » $\left(E C{ }_{17}\right)$; «Il voulut [...] un / reste / moitié / caillou / moitié / prière » $(E C 22 ;$ cf. <editionsunes.fr > à propos d'EC).

32 Nous revenons, en les nuançant autrement, à nos remarques dans la note 6 ci-dessus, sur Bernhard Böschenstein, «Le Méridien et ses matériaux : le centre de la poétique de Celan », op. cit., p. 34, à propos de «l'influence de Mandelstam et de sa conception du poème comme une bouteille jetée à la mer ».

33 Jean-Claude Pinson, Pastoral: de la poésie comme écologie, Ceyzérieu : Champ Vallon, 2020, p. 71. Nous résumons l'essentiel de certaines idées qui s'y trouvent, en adaptant une traduction de la part de Pinson de vers de Mandelstam. Selon ce dernier, «Au peuple il faut un vers énigmatique-maternel, / Que par lui il soit toujours réveillé / Et dans sa vague toute en boucles de lin, châtaigneraie / Dans son souffle se lave » (19janv. 1937, Les Cahiers de Voronej). 
dans Le Troisième lorsque l'Autre semble s'être absenté. Au-delà des dimensions poétologiques, par exemple un désir de pouvoir se situer à tout moment par rapport au cosmos et à la parole, il y a la peur de ne plus avoir accès à l'intime du monde, de ne pas pouvoir l'arpenter, d'être coupé de ses racines en ce qui concerne non seulement les disparus, mais aussi la Nature dans son apparaître à tout instant, sa Phusis, le mystère constitutif qui le sous-tend à la façon du chiffre trois dans l'énoncé «le Troisième». La beauté de la relation que poursuit l'écriture - avec les morts, avec l'Autre, avec l'inconnu du langage et de l'inconscient - reflète la beauté de l'intime du monde, sa matière et ses remuements qui nous font croître, ${ }^{34}$ qu'il faut coudre à soi.

Cinquièmement, Tellermann fait aussi advenir l'intime du monde en employant des vocables et énoncés qui vont dans le sens d'un certain mysticisme. Dans et par le langage, elle coud des fils qui nous relient à l'ici et maintenant. Elle s'occupe non pas d'un au-delà, mais du langage lui-même, de ses lueurs, du «sentiment océanique $»^{35}$ évoqué par Freud, mais qui vient toujours de quelque chose de concret, du mouvement du dire dans le temps et l'espace. On redécouvre que la matière de la langue peut semer « la sensation de l'éternité ». ${ }^{36}$ Les structures langagières que met en œuvre la poésie nous mettent en chemin vers cette sensation, vers cette lumière. Pour peu que l'on se mette à l'écoute, il devient possible de s'en aller « vers / l'infime et / l'étincelle» $(L T$ T), de chercher « ce que les Dieux / avaient ouvert » (LT 18), de faire

34 Ibid., p. 166 : «la Nature comprise comme force qui fait pousser, croître (comme l'indique l'étymologie grecque du mot phusis)». Nous soulignons les dimensions intérieures et extérieures de la Nature, son dynamisme et son mystère. Nous empruntons à Pinson l'image de notre participation « au grand remuement de matière qu'est le monde », ibid., p. 94.

35 Sigmund Freud, Malaise dans la civilisation (1929), texte publié pour la première fois en français dans la Revue française de Psychanalyse 7.4 (1934), p. 692, cité dans FrançoisMichel Durazzo, «Guillevic et l'expérience de la limite», Guillevic : la passion du monde. Actes du Colloque international de poésie les 24 et 25 mai 2002, éd. Jacques Lardoux, Angers : Pu d'Angers, 2003, p. 85-95 (86). Selon l'un des correspondants de Freud, la «source réelle de la religiosité » résiderait dans un « sentiment particulier » qui anime beaucoup de gens, «la sensation de l'éternité », de " quelque chose d'illimité, d'infini, en un mot: d'"océanique" »; « la seule existence de ce sentiment océanique autoriserait à se déclarer religieux, alors même qu'on répudierait toute croyance ou toute illusion ». Selon Durazzo, il s'agit en revanche chez Guillevic de « vide[r] le mot de son rapport à une transcendence pour ne lui conserver que son sens étymologique », et de se mettre en relation alors avec « le meilleur [s]oi-même, c'est-à-dire avec tout l'autre ». Tellermann, pour sa part, semblerait s'intéresser à la fulgurance d'un sentiment océanique à partir de sens ambigus, ambivalents, elliptiques, vibratoires, implicites, qui surgiraient en particulier lors du dialogue qu'instaure le poème avec l'Autre absent. 
« vœu du / commencement » (LT 19), de songer au déploiement de l'âme ( $L T$ 20). Sans qu'il soit question de religiosité, on prend conscience de ce possible. Raison est donnée à la voix humaine « surgie de l'aiguille » du poète «à coup de boucles »; ce sont des boucles sonores, visuelles, textuelles, spatiotemporelles, dont le « rayonne[ment] » apporte un contrepoids aux « sacs / gris » de l'Histoire (EC 28).

Aucune doctrine, donc : seulement l'intuition de ce qui peut échapper à l'intellect, de la richesse et de la profondeur du sol poétique. En cela, l'auteur est en partie redevable à Mandelstam et Celan d'avoir su marcher sur ces terrains, explorer ces seuils, respirer le souffle d'un vers « en boucles de lin, châtaigneraie » dont l'âme peut se vêtir, se nourrir et s'illuminer lorsque l'air et la lumière nous manquent. ${ }^{37}$ Pour combler « la perte de tout ce qui résonne », il faut puiser dans cette «auge invisible » qu'est la parole, dans ses « eaux cuivrées ».38 Dans Le Troisième, l'encre est cette eau, mais qui se met par moments à sécher, bien qu'elle ait alimenté le chant comme prière : «puis soudain / mouvement n'eut / plus d'encre [...] nous avions / mêlé nos prières [...] dans / la langue allemande / je vous ai / attendu » (LT 6o). Si Tellermann évoque ici la langue, c'est de nouveau dans le sens d'un pain à partager avec autrui ou à mâcher seul, de l'écriture synonyme d'un sol plein d'humus, du caractère charnel de la contiguïté des vocables et de l'intime du monde : «la soif / un troisième / dans la bouche / votre encre / / où boire » (LT 78). Sa démarche suit celle de Mandelstam : sa poésie «est un soc qui retourne le temps, en sorte que les couches profondes, le tchernoziom se retrouve en surface $» .{ }^{39}$ Cette terre noire aura sa part de boue, mais celle-ci se fait or dès lors qu'elle est bien labourée.

37 Nous revenons à la traduction citée plus haut des vers de Mandelstam (Jean-Claude Pinson, Pastoral, op. cit., p. 71). Nous traduisons de l'anglais un vers du premier quatrain de ce poème - « The people need light and air » (Au peuple il faut de la lumière et de l'air) -, et le greffons à la traduction de Pinson du dernier des trois quatrains ; cf. Osip Mandelstam, Voronezh Notebooks, op. cit., p. 57 (19 janv. 1937).

38 Osip Mandelstam, Voronezh Notebooks, op. cit., p. 50 (12-18 janv. 1937) : « From what ore will we restore / The loss of all that sounds? [...] the sightless trench, / Full to the brim with coppery water » (Sur quel minerai compter / Pour combler la perte de tout ce qui résonne ? [...] l'auge invisible, / Pleine à ras bord d'eaux cuivrées). Nous traduisons de l'anglais. Cf. EC 40 : « Mots furent notre / auge notre / abri ».

39 Ossip Mandelstam, « Le mot et la culture », De la poésie, op. cit., p. 7-14 (9, 11) : « La poésie est un soc qui retourne le temps, en sorte que les couches profondes, le tchernoziom se retrouve en surface. Mais il y a des époques où l'humanité ne se contente pas du jour présent, soupire après les couches profondes et, comme un laboureur, brûle d'accéder aux terres vierges du temps »; « La vie du mot est entrée dans son ère héroïque. Le mot est chair et pain. Il partage la destinée du pain et de la chair : la souffrance. » Le tchernoziom est une terre noire riche en humus, épaisse et particulièrement fertile (New Oxford American Dictionary, op. cit. ; nous traduisons). 
Tellermann met sa foi dans le mot comme « chair et pain », élément du monde dont le surcroît de vitalité et de vérité n'attend qu'à être relancé dans le monde, activé par le poème, intégré aux sillages des vers. ${ }^{40}$

Qui plus est, Tellermann suit les chemins de la judéité, déjà implicites dans ce que dit là Mandelstam à propos du langage, en s'inspirant de Celan, pour qui compte dans le dépliement de l'infini et l'affrontement avec le néant chaque lettre du dire. Comprendre cet aspect de Celan enrichit considérablement la lecture des recueils Le Troisième et Éternité à coudre quant aux tensions et aux désirs qui s'y expriment. L'idée que le texte - son souffle, ses signes, son histoire, ses couches interprétatives - compte plus que la raison est un trait saillant de la tradition talmudique et kabbaliste auquel Celan s'intéresse de près. Des penseurs tels que Blanchot, Derrida, Jabès et Lévinas, d'ailleurs, tous lecteurs du Talmud, comprennent de cette manière le livre, en tant que socle du devenir, dialogue avec soi et l'Autre, rencontre avec ce que l'écrit voile et dévoile pour que le sens soit de l'ordre de ce que Lévinas appellerait une « caresse » et nous apporte un «pouvoir-être multiple», une « vérité vivante» qui surgit pour chaque lecteur, à l'encontre des synthèses et systèmes. ${ }^{41}$ Chez Tellermann, le texte comme éventail de fils et sensations qui viennent en présence et portent avec eux un sens protéiforme est au cœur du geste de « coudre l'éternité ». C'est ainsi que réfléchir dans Le Troisième à la parole redevient un pas vital qui se fait sur le chemin vers le sacré. ${ }^{42}$

$40 \quad E C$ 81: «Sacrifices / déferlent / en cataractes de / sentences / furent torsion / des univers / murailles / parmi les sillages / furent / l'unique lettre»; EC 89: «Je vous cherchai / au plus bas / de l'âme / là où la langue / retient l'image / et le mot. / C'est vrai / tu as neigé / dans mes mains / des sillages ». À propos de ces « sillages » et de « l'unique lettre » (EC 81, 89), cf. Paul Celan, « Fumée de Pâque », Renverse du souffle, op. cit., p. 147. Voir aussi EC 58 : «j'effleurai votre / mesure / un sillon au cœur / de la figue / / un souffle / qui devient ». Voir Marc-Alain Ouaknin, Lire aux éclats : éloge de la caresse, Paris : Points, 2016, et Le Livre brûlé: philosophie du Talmud, Paris : Points, 2016. Les citations sont tirées des pages suivantes : Éclats p. 38 et p. 61 ; Brûlé p. 230 ; cf. Éclats p. 220.

42 Vocables liés à la parole dans $L T:$ « alphabet» $(26,41)$; « berceuses » $(17,89 ; \mathrm{cf} .70,87)$; « chant » $(17,23,29)$; « cristal » (54); « écriture » $(51,71,82$; cf. 40, 41, 56); « encre » (12, $16,60,78)$; « étincelle » $(7,55,92)$; « fugue » $(17,41,62,69) ;$ « lettres » $(26) ;$ « mot» (69); « paroles » $(26,39,44) ;$ « salive» $(11,38) ;$ « signe[s]» $(20,25,43) ;$ « silences » $(42,69)$; «son» $(42,66,70,76)$; « souffle» $(34,59,64,84,94)$; « syllabes » $(36,69) ;$ « syntaxes » (37) ; « voyelles » $(47)$. Dans $E C$ : « alphabets » $(8,15,54)$; « chiffre » $(14,73)$; « écriture » $(16,17,32,33) ;$ « fil » $(41) ;$ « incendie $[\mathrm{s}] »(9,77,81) ;$ « langue » $(7,53) ;$ « [l] ettre[s] » (12, $43,67,75,81)$; « livre » $(12,72) ;$ « mots » $(7,40) ;$ « papier » $(33) ;$ « parole $[\mathrm{s}] »(7,50)$; «phrase » (9); « plainte » $\left(5^{\circ}\right)$; « rumeurs » $(11,13,54)$; « sentence $[\mathrm{s}] »(44,72,81)$; « signe $[\mathrm{s}] »(6 \circ, 77,81) ;$ « silence $[\mathrm{s}]$ » $(12,13,27,51,59)$; « syllabe» $(9,69) ;$ « tropes » $(68)$; « voix» $(55,73,89)$. Nous soulevons surtout ceux liés à l'écrit. 
En tant que traducteur plurilingue et connaisseur de l'hébreu, ayant d'autres ressources langagières sur lesquelles s'appuyer grâce aux sonorités, à la syntaxe et à la morphologie de l'allemand, Celan peut aller plus loin sur ce chemin, $\mathrm{ou}$, si l'on veut, suivre aisément une bifurcation..$^{43} \mathrm{Il}$ est très conscient, comme les kabbalistes fascinés par la manière dont la recombinaison de lettres crée de nouveaux mots-racines, du pouvoir phénoménologique des mots, du fait qu'ils reprisent sans cesse le possible. ${ }^{44}$ Lorsqu'il parle dans le poème «Quoi coud » du mot qui « avec tout son vert [...] se transplante » et nous encourage donc à le suivre, son mysticisme est foncièrement linguistique, ancré dans la parole, dans la voix qui énonce et qui «coud» le dicible et l'ineffable, la vie ici-bas et l'au-delà. ${ }^{45}$ L'arbre de la vie - soit comme métaphore proverbiale, soit comme image chère à la kabbale des manifestations divines qui descendent du ciel pour se transplanter dans les êtres humains - va donc croître, reverdir, se transplanter dans l'âme pour la déployer, voire rédimer un monde démuni et avili. ${ }^{46}$ Tellermann, pour sa part, est plutôt syncrétiste. ${ }^{47}$ Léblouissement et la jouissance devant les signes comptent autant que l'acheminement vers un Dieu ou des dieux. Or, les mots doivent ordonner un tant soit peu le monde, le ceindre de leur éclat, « tisse[r] / l'air » avec leur « chiffre » (EC 14). Les analyses suivantes auront en filigrane d'autres pistes de lecture possibles qui dépassent le cadre de cette étude, par exemple l'exigence rabbinique de transmettre un «maximum de connaissances » avec un « minimum de mots », l'attention que l'on doit porter à l'« être infinitif » pour résister à tout savoir ou être définitifs, et le caractère sacré et symbolique des lettres dans les civilisations anciennes. ${ }^{48}$

43 Les langues parlées par Celan ou utilisées dans ses poèmes et traductions sont l'allemand, l'anglais, le français, l'hébreu, l'italien, le portugais, le roumain, le russe et le yiddish.

44 Cf. Shira Wolosky, «Paul Celan's Linguistic Mysticism», Studies in Twentieth Century Literature 10.2 (Jan. 1986), p. 191-211 (208). Voir aussi Marc-Alain Ouaknin, Mystères de la kabbale, Paris : Assouline, 2016.

Cf. « Was näht » / «Quoi coud», Partie de neige, op. cit., p. 22-25 (24; nous traduisons). Voir aussi Paul Celan et Gisèle Celan-Lestrange, Correspondance (1951-1970), éd. [et trad.] Bertrand Badiou avec Eric Celan, t. I Lettres, Paris : Seuil, 2001, p. 610-11.

46 Ces remarques doivent beaucoup à Shira Wolosky, « Paul Celan's Linguistic Mysticism », op. cit., p. 201-08.

47 Cf. Aaron Prevots, « Entretien avec Esther Tellermann », op. cit., p. 107 : « Non, je n’ai pas lu la kabbale. Mais j'avais la prétention de rejoindre l'inconscient d'une diaspora. Peut-être la diaspora juive, une autre tout autant à travers elle. Mon père est resté volontairement apatride. Les chiffres, je les aime, ils indiquent le sacré de toute religion humaine, cette façon qu'a l'humain de donner des limites à son angoisse de la mort, comme à sa propre violence, son propre désir de destruction. Il s'agit toujours de sortir du o ou du 1. »

48 Marc-Alain Ouaknin, Invitation au Talmud, Paris : Flammarion, 2001, p. 50 ; Bibliothérapie : lire, c'est guérir, Paris : Seuil, 1994, p. 156 ; et Les Mystères de l'alphabet : l'origine de l'écriture, Paris : Assouline, 1997. Ouaknin est un grand rabbin et spécialiste dans les domaines du 
Plus on s'achemine à côté de Tellermann dans Le Troisième et Éternité à coudre, plus le deuil, le désir et le langage se voient sous un jour nouveau, peut-être celui de «la mémoire / où poussent / de vieux alphabets » (EC 54) ou d'un «Dieu ancien » qui nous « dérobe / l'astre / et le support » (EC 63).

Comment un livre d'amour devient-il une lettre ouverte sur la poésie ? À quel point les discours de Tellermann et de Celan s'entrelacent-ils dans Le Troisième? Quelle sorte de dialogue s'y instaure-t-il ? Quelles en sont les tonalités ? Qu'apprend-on sur cet ouvrage à travers Mandelstam et Celan ? Nous nous proposons de répondre à ces questions à travers des lectures en miroir de textes qui abordent la question des fils du temps à recoudre ou de la parole à repriser. Le départ de ce recueil nous situe au carrefour du deuil et du renouveau. Dans son entretien avec Sophie Nauleau, l'auteur précise que ce livre dédicacé est «le support d'une rencontre», tendu vers un nouvel amour, «mais également vers l'Autre en lui », vers ce qu'on « idéalise » et « qui se dérobe », « cette part d'inconnu qui fait la rencontre et l'impossible de la rencontre ».49 Selon la notice, de même, « [i]l y a deux livres dans Le Troisième » : d'une part, «les premiers mots, le secret tu, la trace du poème sur la page, comme une cicatrice, une incision. [...] Le secret de l'Autre et l'impossible jusqu'à lui »; d'autre part, un recueil « en éclatements successifs, en ouvertures - incisions - jusqu’à l'intenable », jusqu'à planter « des fleurs de cailloux sur les tombes ».50 Certes, on comprend sans peine le "désir de fusion vers un "un" en l'Autre qu'on admire $» .{ }^{51}$ Les nombreuses références à Celan dans Le Troisième font de lui celui que la locutrice a attendu «dans / la langue allemande » (LT 60). Telle sera notre hypothèse dans l'analyse suivante, où notre double objectif sera de mieux comprendre le mot-symbole « le Troisième » et la poésie poétologique des deux auteurs.

Commençons par donner le ton: celui d'un «vibrato douloureusement muet $», 52$ non loin de ce même vibrato chez Mandelstam ou Celan, allant de

Talmud, de la kabbale et de l'histoire du langage, notamment en ce qui concerne des liens à la pensée française contemporaine.

Sophie Nauleau, «Entretien avec Esther Tellermann », op. cit.

50 «Esther Tellermann : Le Troisième », <editionsunes.fr >.

$51 \quad$ Sophie Nauleau, « Entretien avec Esther Tellermann », op. cit.

52 Paul Celan, «La poésie d'Ossip Mandelstam », op. cit., p. 11. Cf. Paul Celan, Le Méridien \& autres proses, op. cit., p. 112, où Launay opte pour la traduction «vibrato de douleur muette » (l'auteur souligne). 
pair dans ce cas-ci avec l'envie d'être moitié dans le rêve, moitié dans le réel, de « retourn[er] vers / l'Est / et dans l'humide » (LT 8), de descendre jusqu'aux « mondes de dessous » ( $L T$ 11) et de creuser ainsi lélémentaire dans sa dimension régénératrice. Ce que Celan dit sur Mandelstam s'y applique bien : il y aura un « rapport de tension entre les temps, du propre et de l'étranger ${ }^{53} \mathrm{En}$ un mot, il y aura sans cesse du relationnel. Ces rapports vont s'inscrire dans la forme tellermannienne. Vis-à-vis de Mandelstam, nous considérerons notamment la compacité du poème ; les rapports entre parole et histoire ; la reprise de vers dans un poème adjacent ou similaire ${ }^{54}$; et la nomination relativement plus abstraite que chez Mandelstam, qui aime à faire s'entendre et se voir dans ses descriptions détaillées l'intime à portée inouïe et imprévisible des heures qui passent, par exemple l'Eucharistie comme soleil d'or qui fait penser aux «syllabes grecques» et donc au «monde entier tenu dans les mains comme une simple pomme $» .55$

Souvent, le vibrato de douleur dans Le Troisième vient de l'entrecroisement dans l'esprit du lecteur des incisions, des motifs inattendus, de tout ce qui jaillit sur les chemins de l'inconscient, que ce soit l'« accord des tourbillons » (LT 7) emblématique de la poésie, ${ }^{56}$ «le jaune du / juif » et «l'ordre / du fou » (46) qui font penser à la Shoah ou des «berceuses pour / notre marche» (89) qui suggèrent un retour momentané au calme. En plus des mots ayant un « pouvoir rayonnant $»^{57}$ non loin de ce que l'on peut remarquer dans ceux de Mandelstam, force est de constater la douleur qui en ressort chaque fois que Tellermann se charge de porter celle de l'Autre, de s'identifier à sa « cendre » et ainsi de s'emplir « de noirceur et / d'orages » (10). Alors que le lecteur de Mandelstam pensera forcément à la façon dont les choses entrent en rapport, par exemple les années ou les ères, celui qui aborde Le Troisième songera à «cet être-ensemble ${ }^{58}$ au plan singulièrement humain, à la manière dont les êtres entrent en rapport. En voici plusieurs sens profonds du titre : rester, comme le

53 Ibid.

54 Osip Mandelstam, The Selected Poems of Osip Mandelstam, trans. Clarence Brown and W. S. Merwin, New York : NYRB, 1973, p. 54.

55 Ibid., p. 30. Pierre, poème 117 [1920]. Nous traduisons de l'anglais.

56 Sophie Nauleau, « Entretien avec Esther Tellermann », op. cit. Tellermann en parle.

57 Philippe Jaccottet, « Quelques notes à propos de Mandelstam », Une transaction secrète : lectures de poésie, op. cit., p. 172-82 (176) : « Chacun des mots des poèmes, employés directement ou sous forme d'image, [...] est chargé comme un astre de pouvoir rayonnant, chargé d'expérience profondément vécue ou de rêve profondément rêvé. »

58 Paul Celan, «La poésie d'Ossip Mandelstam », op. cit., p. 11 : « Les choses entrent en rapport », mais il s'agit d'un « être-ensemble » où reste ouverte « la question de leur provenance et de leur destination, [...] une question [...] qui indique le chemin de l'ouvert, de l'investissable, du vide, de l'espace libre ». 
précise Celan à propos de Mandelstam, « dans une ouverture temporelle, [où] le temps peut s'y adjoindre, le temps participe ${ }^{59}$; se situer, ce faisant, à la faveur d'images assez immédiates, dans l'intersubjectif, l'échange, la rencontre poétiquement charnelle avec l'Autre et avec la parole en tant que demeure, profitant des miettes d'heure pour soulever avec douceur des liens. Tel est le sens des vers suivants, qui font écho à l'idée celaniennne d'« heures qui se brisent en miettes ${ }^{60}:$ « Nous serons 3 dans les calices [...] dessous encore / sont les miettes où / nouer nos / formules / je m'allongeai / sur toi / te confondant / à l'encre » (12). Rien de plus épistolaire, d'ailleurs, que cette façon de «nouer » les formules, d'être ensemble à travers l'écrit, de se réunir dans la parole.

Le ton varie en fonction des mots choisis et de l'humeur de celle qui écrit. La polysémie fait partie de cette variation, par exemple ces «calices» qui convoquent le vin et l'Eucharistie ainsi que le sépale en botanique. Lire ces poèmes à travers Celan renforce l'idée que Tellermann veut «empli[r] l'espace » de sa fugue, entendre un chant qui appartienne également à elle et à lui, à ceux et celles que chacun souhaite tisser dans la trame langagière : «Avec des noms / je voulais / vous continuer / entendre moitié / de chant» (17). Le mot-symbole «le Troisième» renverrait donc au nouvel espace que crée son dire lorsque ces chants s'entrecroisent, au nouveau possible d'une fugue « assourd[ie] » (62), lissée, apaisée, en aval des fleuves réels ou métaphoriques assombris par la mort, des larmes déjà versées. Cela dit, il est intéressant de voir l'équivoque de nombreux vers, en quelque sorte un aspect du vibrato tellermannien. Ses «valses lentes » $(15,21,25)$ font contraste avec le tango fou mené par les maîtres des camps que critique Celan, ${ }^{61}$ mais s'accompagnent tout de même de «cris», de «brûlures», d'« étoiles». On peut remarquer avec Tellermann combien cet entretissage symbolique d'énoncés polyvalents, qui comprend la superposition du dire d'époques diverses, ajoute à l'infini de la parole de nouvelles couches de sens et de sensations, telles ces «terres

$59 \quad$ Ibid., l'auteur souligne.

6o Paul Celan, « Devant une bougie », [De seuil en seuil], Choix de poèmes, op. cit., p. 97-99. Cf. $s N$ 64 : « votre bouche à ronces / pareille à la / mienne / miettes d'heure / à prononcer. / Je vous entourais / de formules / au milieu de / la roue / nous nous comptions / en un ». Gardons en mémoire l'image de « La Treizième » dans « Artémis » de Nerval. Voir aussi $c E$ 19 et 88 : «Dans le nom du / troisième et sa boucle / j'emplissais le monde / d'épilogues »; « tu as scellé en elle / les versets / viennent à chacun / les lisières des bords / d'un horizon / loin devant / cheveux dénoués pour les orages / / le troisième / ayant nom du premier ».

$61 \quad$ Ibid., p. 52-57 et p. 332nı. Avant de figurer dans le recueil Pavot et Mémoire en 1952, le poème « Todesfuge » (Fugue de mort), écrit en 1945, paraît en revue dans une traduction roumaine de Petre Solomon, un ami de Celan, sous le titre « Tangoul mortii » (Le tango de la mort). 
blanches » que sont les pages qui restent à écrire et ces « océans de cuivre » qui surgissent lorsqu'est atteint « l'autre rive » $(15) .{ }^{62}$

La manière dont Tellermann bâtit un Nous ${ }^{63}$ amplifie ce sentiment océanique. Elle insiste sur ce pronom et sur des actions faites à plusieurs, même si l'identité de ce Nous doit rester inconnu, secret, énigmatique. Le mystère de ce Nous fait retentir diverses résonances, de sorte qu'il corresponde autant à une relation amoureuse quà une relation poétologique, avec les chemins vers l'Autre en nous-mêmes qu'ouvre la langue. Les références au chiffre trois ont pour but de nous relier au dehors et au dedans, au devenir des mondes extérieur et intérieur, et à la parole comme écho de ces forces créatrices. Dans le flux et le reflux du dire, le trois fait surface comme union à la fois ressentie et anticipée. ${ }^{64}$ Même si le mot-symbole «le Troisième » doit rester ambigu pour garder sa vigueur, de nombreux énoncés nous offrent des indices quant à sa profondeur et son rôle de principe structurant. Être ensemble, c'est déjà coudre à soi l'éternité. Regardons quelques exemples. Notons l'union souhaitée, ainsi que la présence de Celan dans le tissu allusif.

De manière générale, le Nous est un horizon, une porte, un seuil, un point de mire dans le dialogue avec soi. À deux ou à plusieurs, on fait mieux partie d'un tout: des « 3 saisons » (13), des « musiques et [d] es / cendres » (14), des « 3 univers » (20), des « Parnasse » (25). Vouloir, c'est déjà presque pouvoir, pourvu que l'on soit accompagné. C'est dans ce sens que va le dire oraculaire. Si la barque poétique se charge de « toutes les palmes » (13), si « les bords sont / les océans » (27), c'est moins pour compenser la mélancolie que pour constater ce dont on est capable et souhaiter qu'aient lieu des commencements. Dans les vers « Nous / jetions dans nos / silences / voulions déployer / lâme / une corde

62 Il y a aussi, au plan symbolique, le rythme ternaire et donc lié au Troisième qui est introduit par la valse comme morceau de musique à trois temps et donc fait pour nous soulever quoi qu'il arrive. Il y a d'ailleurs une dimension plus humaine - tantôt mélancolique, tantôt enjouée - de cette danse vis-à-vis de la « Fugue de mort » celanienne plutôt funèbre.

63 La présente analyse ne s'appuie pas sur Irigaray, mais le syntagme «bâtir un nous » vient d'elle (l'auteur souligne). Voir Luce Irigaray, J'aime à toi, op. cit., p. 173. Il s'agit chez Irigaray d'une lente transformation qui a lieu dans le respect absolu de la singularité de l'Autre, dans un esprit de dialogue qui permet à la fois de mieux percevoir et d'évoluer ensemble.

64 Cf. Nanon Gardin et al., Petit Larousse des symboles, op. cit., p. 626-27 : « Chiffre de l'union et de la perfection, le trois représente l'ordre du cosmos. [...] Trois est le nombre parfait, l'image sensible de la divinité. » Gardin évoque par exemple Aristote à propos du temps et des trois dimensions (largeur, longueur et hauteur); Pythagore à propos du début, du milieu et de la fin ; Platon et les trois parties du corps (tête, tronc et bas du corps); saint Paul quant à l'esprit (pneuma), à lâme (psyché) et au corps (sôma); le chiffre trois désignant dans les religions du Livre le monde terrestre, l'enfer et le paradis ; et le trois comme chiffre sacré dans la création. 
tendue sur / les 3 univers [...] allions / au-delà du / signe et des / alliances » (2o), le fil poétique est solide, robuste, plein de couleur et de promesse. La découverte de soi et de l'Autre se fait éthique, la solitude une disponibilité, une réceptivité. Le lent dépliement de la parole permet d' «enlac[er] / l'obscur et / les splendeurs » en soi, dans le monde et dans nos relations (14), fait goûter « la ferveur » (25) synonyme d'une foi en la parole poétique. De même, une série de verbes au futur souligne cette idée de croyance, d'espoir en l'avenir. Les énoncés abstraits et allusifs, ainsi que le caractère énigmatique du Nous, ont un effet berceur, exaltent l'amour, l'amitié et la parole en tant que pont du passé vers cet avenir :

Nous habiterons / des maisons de sable [...] détisserons / les oracles / à chaque fois / plus nus / rejoindrons / l'innocence / délivrerons / les captifs (30)

Ou bien / retournerons aux / dieux afin qu'ils / désaccordent / la splendeur [...] saurons-nous / saisir sous les / océans / les fontaines ? (31)

Baudelaire, Hölderlin, Hugo, Mandelstam, Perse, Reverdy, Trakl : on peut imaginer à loisir quelles sont ces maisons de sable, quelle «terrible / musique » sera «attest[ée]» (28). Il s'agit plus de frontières floues entre le dire d'autrefois et celui d'aujourd'hui, d'un feu de signes (25), que d'actions précises pour changer l'humanité. Il faut avant tout demeurer dans «l'innocence» (30) d'un Nous, d'une totalité, de relations légèrement « désaccord[ées] » (31) pour mieux durer.

Les allusions à Celan approfondissement notre compréhension du recueil $L e$ Troisième largement. Bornons-nous en pour l'instant à trois : les « anneaux », les «septembre » et les «mains ». Relevons aussi combien le vers saccadé, le mot isolé et le mélange d'ardeur et de pauses méditatives font penser à lui, aux disparus auxquels manque une parole pleinement habitable, à ceux et celles qui cherchent «là-bas / une / fois encore / à fleurir » (18). Revenons surtout à l'idée de «deux livres dans Le Troisième », ${ }^{65}$ l'un amoureux et l'autre plutôt hanté par la présence de Celan, voire de la mort et du souhait d'aider autrui à renaître à la parole. Ressortent ainsi les nombreux écueils quant au désir d'enlacer un devenir commun. Les vers suivants de Tellermann servent de contrepoids à cette douleur, tout en l'intégrant, la réorientant: «[Avais-je assez] enserré les paysages / dans les anneaux / pour vous construit les / tombes

65 « Esther Tellermann : Le Troisième», <editionsunes.fr>. 
déposé / au long de Toi / les énigmes / [...] épuisé votre / / encre ? » (16). Là où Celan doit creuser obstinément la terre de la parole, « [s]e creuse vers » un Tu pour que «l'anneau » prenne forme, ${ }^{66}$ Tellermann s'ouvre à une sensation de l'éternité, à l'acceptation des cycles de mort et de vie. C'est comme si elle lui renvoyait son dire, certes sous une forme plus rassurante, mais aussi en respectant celui-ci profondément, avec des gestes d'amour comme le suggèrent les images « enserré les paysages / dans les anneaux », « construit les / tombes » et « déposé [...] les énigmes ». La dernière image, « épuisé votre / / encre », va de pair avec les actions dépeintes quelques pages plus loin : «je t’avais / dessiné comme / on ouvre la grille / enchevêtré / d'anneaux » (23). Elle semble vouloir tout faire pour cet Autre, d'où son inquiétude lorsqu'elle a peur d'avoir perdu le contact avec lui et « déf[ait] / la roue » (32), d'avoir trop voulu établir des alliances et donc rompu les fils de lumière dont devaient témoigner ses propres poèmes. Les divers mots de passe - anneaux, tombes, énigmes, encre, roue sertissent le dire celanien, désignent le relationnel avec de grands égards.

Il en est de même à propos des « septembre » et des « mains ». Une remarque dans tel ou tel vers qui n'a l'air de rien, ou qui est assez énigmatique, peut tout à coup étinceler. Regardons trois textes :

C'est encore notre / voyage comme / force close et / votre sourire / depuis la profondeur / peut-être voulions-nous [...] attendre les septembre et / les songes pour / mourir dans les / 3 saisons (13)

Ou bien / pourrions être / légers ouvrirons / les septembre et / nos doigts dans / la moitié / de chant (29)

Car étions / au-delà greffions / aux nuages / des airs / dans l'entrée de / septembre avions / continué / la voix / serons-nous encore / avec la neige / à entendre / derrière nous / brassées d'étoiles / saturées de / paroles? (39)

Ces vers peuvent se lire sous le signe de l'exigence celanienne de se tenir debout. Le doucement élégiaque renvoie en même temps à de profondes difficultés d'être, à l'ampleur de la tâche du poète. Il faut soigner la parole, avoir en esprit le Nous, intégrer d'autres voix, rester à l'écoute de la « voix » (39) tant $\mathrm{du}$ dehors que des disparus. Pour Celan, comme pour Tellermann marchant dans le sillage de celui-ci, cela veut dire aussi se pencher sur le néant, chercher

66 Paul Celan, « Es war Erde in ihnen » / « Il y avait de la terre en eux », La Rose de Personne, op. cit., p. 10-11 (10; nous traduisons). 
un abri. ${ }^{67}$ La problématique du «Troisième » - de l'élan vers l'Autre, du désir de tisser des liens avec celui-ci, du devoir éthique et des rapports renouvelés au cosmos qui en résultent - rejoint donc celle de l'aveuglement. Le poème ne peut être offrande que si le poète accepte les moments de détresse, de perte ${ }^{68}$ les septembre, quand les feuilles tombent de l'arbre poétique après des saisons mouvementées, et qu'il faudra apprendre à voir à travers le vide et le néant qui commencent à troubler la vue et l'ouïe. Est aveugle celui qui laisse advenir ses quelques mots « de pur / automne et soie et néant», ${ }^{69}$ qui sait rester réceptif au chant comme souffle, à sa propre «moitié / de chant » greffée à ce souffle $(29,39)$ et à la part d'inachevé qui s'y trouve. Comme le dirait Mandelstam, c'est aussi le lecteur aux « doigts voyants $\gg^{70}$ qui se fait aveugle, acceptant l'offrande faite de ces pleins et vides, la parole dénuée d'effets et de bavardages qui rompraient nos liens avec le réel. Il faut ce discours autre - très palpable, hautement vécu, intensément partagé, inscrit dans le temps et proche de l'indicible - pour tendre la main à l'Autre. ${ }^{71}$ Déployer l'âme exige de mieux connaître celui-ci en tant qu'absence à laquelle la parole nous fait accéder.

En effet, Le Troisième nous ouvre au temps : celui du dedans des mots ou des pensées de la locutrice, celui moins intime de l'événement. Il s'agit d'une douleur profondément ressentie vis-à-vis de l'Autre et de relations qui se développent. Les énoncés elliptiques et allusifs nous font ralentir le pas, traverser des moments passés, présents ou à venir. L'expression « halo de nuit » (LT 34) est particulièrement révélatrice. Lue au travers du texte «Mapesbury Road»

67 «Esther Tellermann : Éternité à coudre», <editionsunes.fr> : «Livre posé au bord de la disparition, Éternité à coudre se déploie comme une parole rituelle, un exorcisme. Penché sur le néant, cherchant un abri dans les mots et les corps, quelque chose de l'ordre de la permanence du monde dans les cendres, dans les noms brûlés, les noms mâchés. » Comme Le Troisième et Éternité à coudre composent un diptyque, il est possible que ces propos s'appliquent aux deux recueils.

68 Cf. Paul Celan, «Odeurs d'automne muettes », [La Rose de Personne], Strette \& autres poèmes, op. cit., p. 47 : «Une perdition étrangère prit / corps là contre, tu aurais / vécu / presque.»

69 Paul Celan, «Erblinde» / « Sois aveugle », Renverse du souffle, op. cit., p. $72-73$ (72; nous traduisons).

70 Ossip Mandelstam, «Le mot et la culture», De la poésie, op. cit., p. 7-14 (12) : «Écris des vers sans images si tu le veux, si tu le sais. L'aveugle reconnaîtra le visage bien-aimé, l'effleurant de ses doigts voyants, et des larmes de joie [...] jailliront de ses yeux après une longue séparation. »

71 Nous condensons diverses références. De Celan, voir par exemple «La main pleine d'heures », «Le festin », « CEil sombre en septembre » et « En voyage », Pavot et Mémoire, op. cit., p. 25 , p. $45^{-47}$, p. 49-51 et p. 93 ; Grille de parole, op. cit., p. 9-13, p. 49-51, p. 65 et p. 83 ; La Rose de Personne, op. cit., p. 25, p. 37, p. 71 et p. 131-33 ; Renverse du souffle, op. cit., p. 11, p. 23, p. 49, p. 53, p. 71 et p. 173 ; et Partie de neige, op. cit., p. 21 et p. 159. 
de Celan, où il est question d'un "plein / halo de temps », ${ }^{72}$ l'idée d'un « halo de nuit » (LT 34) s'approfondit. D'une part, ce poème de Celan traite de façon oblique d'incidents violents. On apprend qu'un coup de feu peut faire un trou dans le temps et signaler la durée d'une forte souffrance, l'impact sensoriel d'une «demi-horloge marquant les heures magnoliennes » qui éclosent dans la mémoire, indiquant qu'il ne faut rien « ajourne $[\mathrm{r}] \gg{ }^{73}$ Le Troisième traite de la durée d'une souffrance moins violente, celle de ne plus avoir un accès adéquat à une vérité de parole. À l'instar du poème de Celan, ${ }^{74}$ les heures y sont magnoliennes, dans le sens d'une attente de jours pleins de force et de sève, d'éventuelles floraisons printanières odoriférantes malgré le passage par une saison poétiquement creuse : « tu écrivais [...] dans le cru / du magnolia » (LT $42)$; « Reste et mapprends / les magnolias [...] voix / sur la voix / emplie de voix / qui me séparent / donnent assez / d'ombre» (LT 95). Pour Tellermann, le poème sera plein lorsqu'il se remplira de nouveau de voix, de relations humaines entrelacées dans celles que nous entretenons avec le cosmos et la mémoire.

D'autre part, l'emploi dans «Mapesbury Road» du mot Zeithof (halo de temps) résonne dans ce contexte au niveau conceptuel, tel ces arbres qui convoquent la mémoire et l'oubli, les rapports avec soi et la relation avec autrui. Selon Jean-Pierre Lefebvre, Zeithof est un concept « créé par Husserl », une expression qui «signifie littéralement "halo de temps" ». ${ }^{75}$ Celan charge ce concept d'une dimension historique, d'un sens «politico-poétologique ».76 Il voudrait que le lecteur reconnaisse les allusions potentielles, prenne en compte aux niveaux affectif et linguistique le «halo lunaire $»^{77}$ recouvrant un énoncé, considère dans la vie réelle les vérités qu'englobent ses références énigmatiquement condensées. Le travail poétique doit exiger ce travail de la part du lecteur. Ainsi la référence de Tellermann à un « halo de nuit » pourrait-elle se lire en lien étroit avec par exemple les persécutés - y compris Celan - dont la « cendre » devient la sienne ( $L T$ 34). Bien que Tellermann évite de parler d'événements ou d'épisodes historiques, les mots clés qu'elle emploie pour suggérer ce qui la préoccupe peuvent être significatifs, d'une portée océanique, pourvu que l'on suive leurs fils sémantiques.

\footnotetext{
72 Paul Celan, « Mapesbury Road», Partie de neige, op. cit., p. 64-65 (64; nous traduisons).

73 Ibid.

74 Ibid., p. 194. Celan voit les magnolias de Londres et pense aussi à ceux de Czernowitz, sa ville natale, dont il reste cruellement séparé.

$75 \quad$ Ibid., p. 194nu1.

76 Ibid., p. 195 .

77 Ibid., p. 195 .
} 
Par ailleurs, comme le suggère Celan à propos de Mandelstam, les cadences et sonorités d'un poème participent à cet effet de «halo ${ }^{78}$ Le halo temporel au-dedans du poème coïncide avec celui plutôt historique qui est évoqué. Dans ce cas-ci, l'isolement de mots et de phrases nous fait parcourir le chemin historique dont il est question. ${ }^{79}$ Dans les vers « je n'eus pas assez / de larmes et de / secondes » (LT 34), le mot isolé « secondes » appelle le temps passé à pleurer les peines qu'ont subi Celan et d'autres. Dans les vers suivants, « [À] l'intérieur de / toi avais-je laissé / ma cendre / halo de nuit / fraction / d'aube et / de souffle ? » (LT 34), l'alternance entre des consonnes sourdes et sonores - $[\mathrm{t}]$ et [d], [f] et [v] - souligne de telles durées. La « cendre » doit se transformer, par le poème, en « fraction / d'aube et / de souffle », autant par le passage des années que par l'adoucissement sonore que privilégie ce vers. Même si pour l'instant la «pénombre » (LT 34) affective ne disparaît pas, la volute de mots nous fait revivre la relation poétologique de Tellermann avec Celan. C'est un dire où seule la relation poursuivie peut mettre le Nous au « centre / du signe », « en son plein / halo » (LT 43), là où les mystères du temps rejoignent ceux du «mourir» $(L T 13,42)$.

Dans Le Troisième, ce drame ne se dénoue pas. Peut-être ne le faudrait-il pas, afin que nous comprenions à quel point ces poètes peuvent être des « sœur[s] en mourir » ( $L T$ 48). La mise en relief des hauts et des bas de cette relation nous montre sa fonction réparatrice : son immédiateté, sa nécessité, le besoin d'accéder aux profondeurs de la parole où trouver «l'épaisseur du sensible » ( $N N$ 34). Les mots ouverts par ce livre-poème sont «infléchi[s] par la perte » $(N N 38)$. Or, cette perte se dédouble. D'une part, Celan ne cesse de parler pour les disparus. D'autre part, perdre brièvement Celan comme «sœur en mourir », c'est ajouter des abîmes à une parole déjà trouée, et ce, au risque de voir s'effacer certains traits du récit mythique sur la genèse lente du « lœss » et de nos « exils » $(L T 74),{ }^{80}$ de limiter l'accès au « rien » fondateur qui veut toujours

78 Paul Celan, «La poésie d'Ossip Mandelstam», op. cit., p. 11 : « C'est ce rapport de tension entre les temps, du propre et de l'étranger, qui confère au poème mandelstamien ce vibrato douloureusement muet à quoi nous le reconnaissons », vibrato qui est «partout, dans les intervalles entre les mots et les strophes, dans les "halos" où se tiennent les rimes et les assonances, dans la ponctuation ».

79 À propos de l'historicité, contrepoids de l'hermétisme, même si les deux caractéristiques vont parfois de pair, cf. Shira Wolosky, « The Lyric, History, and the Avant-Garde : Theorizing Paul Celan », Poetics Today 22.3 (Fall 2001), p. 651-68. Voir aussi Frédéric Marteau, « Contra-diction. Paul Celan et l'art du contrepoint », op. cit., p. 64-65.

$80 \quad$ Cf. Paul Celan, « Poupée de lœss », Partie de neige, op. cit., p. 119. Le lœss est un limon très fertile, une roche sédimentaire détritique d'origine éolienne. Cette poussière dispersée par le vent recouvre en Europe de vastes surfaces et peut avoir des formes singulières. Voir p. 23ını à propos de « poupées de [ou du] lœss » comme concrétions calcaires dont la 
« pousse $[\mathrm{r}]$ » $\left(L T\right.$ 57) ${ }^{81}$ À force d'évoquer l'infime, Celan personnifie le « Reste chantable », être dont le profil est une «masse de regard » et vers lequel dérive le « cœur-satellite» du cosmos, lui montrant qu'il arrive toujours, «à proximité », quelque chose à raconter. ${ }^{82}$ C'est une sorte de lueur minuscule dans un monde dystopique hanté par la mort, une mèche qui provoquera de nouveaux incendies, un écho de « La Treizième » qui illuminait le cosmos nervalien.

Cependant, Le Troisième introduit aussi des nuances saisissantes. Il ne traite pas que de ce reste. Il y a des abstractions introduites de manière à approfondir les problématiques principales concernant le temps et le fil de la parole qu'il faut coudre, ainsi que des descriptions d'actions liées à une relation à deux. Par exemple, il faut à tout prix parler, sinon «temps s'arrête » (48). De simples morceaux « de symbole / et d'étreinte » (52), telle la pomme de Mandelstam citée plus haut, nourrissent le dialogue entre soi, le monde et l'Autre. À l'instar du leitmotiv «nager [ensemble] » de Celan, expression du désir de voir la parole reverdir et se renouveler, ${ }^{83}$ ce dialogue se fait relation tour à tour intime et charnelle. Coudre des fils de parole doit faire fleurir en l'Autre « les / métamorphoses » $\left(L T 5^{2}\right)$. Le Je et le Tu se nouent (58). Lorsque le Tu se transforme en Vous (62), c'est comme si ces métamorphoses avaient eu lieu. Coudre une éternité à «nos deux noms » les rendra plus habitable, une demeure où être en «chacun » (61). La parole incarne un sentiment océanique que font étinceler le mot, le geste, le pas fait vers autrui. On apprend les dangers d'une étreinte du temps trop serrée, le vertige qui nimbe le désir de vouloir «nommer l'inouï du Un » ( $N N 34)$, le rôle primordial de « la lumière » (LT 64) qu'apporte la relation et du «son» en tant que lien provisoire avec la mort (66). Le Je et le Tu du poème descendent « sous / les siècles » (67), celui-ci en tant

genèse correspond au poème celanien - probablement aussi aux tensions entre le lyrisme du passé et la contre-parole qui doit s'élaborer pendant l'après-guerre. Notons également la notion de sœurs en mourir ; cf. Paul Celan, «Strette », [Grille de parole], Strette \& autres poèmes, op. cit., p. 25-39 (25).

81 Cf. Paul Celan, «Décapé », Renverse du souffle, op. cit., p. 49, où le poème celanien se heurte au 'rien' du lyrisme moderne, au vide inhospitalier du langage des bavards, et doit absolument aller plus loin, trouver des cristaux de souffle qui pourront plutôt témoigner.

82 Cf. Paul Celan, «Singbarer Rest» / «Reste chantable», [Renverse du souffle], Choix de poèmes, op. cit., p. 242-243 (242; nous traduisons) : «Reste chantable - silhouette / de celui qui silencieusement / à travers l'écriture-faucille a percé ». Voir aussi les commentaires de Jean-Pierre Lefebvre, p. 215n1, vis-à-vis des liens entre cet être et l'Histoire.

83 Cf. Paul Celan, « Par deux » et « Toi aussi, parle», De seuil en seuil, op. cit., p. 45 et p. 105 ; «Avec lettre et horloge », Grille de parole, op. cit., p. 19 ; « Tübingen, janvier » et « Les syllabes douleur», La Rose de Personne, op. cit., p. 39 et p. 131-33; et «Par les rapides de la mélancolie», «Port» et «D’avoir vu les merles», Renverse du souffle, op. cit., p. 19, p. $85^{-89}$ et p. 163 . 
qu'«icône » peut-être figée dans une relation symbolique (67), mais aussi ce qui « enfant $[\mathrm{e}]$ » pour que ces recherches perdurent (66), pour que l'on continue à nager, à marcher ensemble (89), à «s'incline[r] pour [...] entendre » les morts ( $N N 45)$. L'imparfait de bon nombre d'énoncés peut mettre une distance entre une action et son éventuelle reprise, mais nous voyons néanmoins qu'il est capital de poursuivre : « Nul ne / cherche / à côté d'elle / les flocons / Ophélie noire / sortie des fosses » $(L T$ 87); « pourrions-nous / sans nous nous / faire verbe / ouvrir les océans / nous donner des / secondes encore / couche après couche / élimer les guerres [...] être assez aveugles / / pour devenir » (LT 91). ${ }^{84}$

Pour Tellermann, Celan est emblématique du besoin de rebâtir la parole afin d'accéder au monde de l'Autre, de pouvoir authentiquement s'adresser à lui. Il réunit en lui d'autres âmes sœurs de la poésie, d'un Est imaginaire ou rêvé dont la boue est particulièrement riche en or, où l'on peut, comme le dirait Mandelstam, aborder de front notre « destinée ${ }^{85}$ ainsi que l'histoire de nos gestes vers le sacré. Gardons en esprit ce qui est dit dans Le Troisième sur cette figure-silhouette qui ouvre un espace «plus bas encore» (70) où Tellermann peut la chanter, et, ce faisant, déployer de nouveau « l'âme » (20), coudre à soi l'intime du monde, faire entendre d'autres morts, montrer qu'il reste toujours possible de mêler « nos / neiges » (90) et de persévérer dans l'échange de la parole, ainsi que de suivre des chemins de l'inconscient plutôt individuels. On verra dans l'analyse suivante comment le poète propose de retrouver sa voie/x; quelles découvertes ont lieu lorsque Tellermann parvient à accueillir l'énigme ; quelles présences oniriques se mettent à rôder lorsqu'il est question de recoudre nos liens avec les disparus ; l'apport du féminin à la prière pour les morts ; et le rôle que joue Celan dans cette inscription des seuils.

\section{Déployer de nouveau l'âme : Éternité à coudre}

Reprenons la notion de « coudre » léternité. Geste solitaire, d'abord : accueillir les fils d'émotions, d'images, de pensées et sensations qui viennent en présence; contempler leur sens protéiforme ; suivre la marche du temps pour mieux faire partie du cosmos; se laisser porter par le courant des mots, leur

\footnotetext{
84 Cf. Paul Celan, «Wortaufschüttung / « Remblaiement de mots », Renverse du souffle, op. cit., p. 44-45, à propos du témoignage qu'opère le poème à travers des mots qui se mettent lentement en place sous forme de dépôts volcaniques et qui rétablissent ainsi l'ici et maintenant sur la terre. Voir aussi p. 209nı, ainsi que dans John E. Jackson, Paul Celan: contre-parole et absolu poétique, op. cit., p. 129-30, la traduction citée plus haut, « Levée de mots, volcanique».

85 Ossip Mandelstam, De la poésie, op. cit., « Le mot et la culture », p. 7-14 (11).
} 
soie, leurs distances, leurs cadences, leur mise en bouche ; aspirer, expirer, transcrire des traces de monde, de souffle ; ausculter, innerver, assurer la circulation sanguine, insuffler la vie au réel ; «déplac[er] les bornes [...] de la mesure du temps » ${ }^{86}$; infléchir ce souffle, ce temps, ce réel, cette vie. Geste intersubjectif aussi : s'orienter vers l'Autre, mettre le signe en mouvement vers lui ; enraciner dans la parole les disparus, ceux et celles poussés vers ses marges ; accepter d'être déraciné par ces actions, d'être porté vers le passé, les persécutés et le « dedans » de la parole (EC 41) qui nous est offert. Enfin, geste sacré : «appren[dre] / l'absence », «modul[er] / un Dieu ancien » qui en se cachant nous « dérobe / l'astre / et le support » (63); faire alliance avec les disparus, battre la mesure poétique en marquant le rythme de leurs pas, les revoir pour qu'il y ait échange de serments, de mots raclés, de dignité. ${ }^{87}$

Ces derniers aspects seront particulièrement pertinents lors de l'argumentation de ce deuxième volet du diptyque. La parole rituelle de ce recueil se penche plus sur «l'énigme» sans laquelle une langue «agonise » (7), sur la folie du monde violent ${ }^{88}$ et sur le néant de la parole celanienne - vide hospitalier non sans étincelles et lueurs, écoute de l'Autre et désir de réconciliation - que sur l'intime d'une rencontre amoureuse et le temps désaccordé. À travers les énoncés et leurs trames allusives, la dimension historique est aussi présente que la dimension poétologique. Accepter la boue, comprendre « [q]uoi [...] enlise nos / paroles » (50), « défaire / l'épaisseur » (53), c'est aussi faire un travail de mémoire très douloureux. Se déploie de nouveau l'âme lorsque Tellermann fait non seulement, comme Celan, un travail de mémoire «médiatisé » par les «silences successifs » de l'écriture poétique, ${ }^{89}$ mais aussi son propre

86 Cf. Yves Boudier, « [note de lecture] Esther Tellermann, Le Troisième », Poezibao, 31 mars 2014, < poezibao.typepad.com>; voir aussi Yves Boudier, « Esther Tellermann : Éternité à coudre », CCP : cahier critique de poésie 34.2 (juil. 2017), <cahiercritiquedepoesie.fr >.

87 Cf. Paul Celan, « Avec les persécutés », Renverse du souffle, op. cit., p. 37, ainsi que p. 206ng.

88 Nous pensons à Sylvie Germain évoquant la « folie du monde bavard et violent » et les éblouissements discrets qui y font contrepoids. Voir Sylvie Germain, Songes du temps, Paris : Desclée de Brouwer, 2003, p. 22. Notons aussi vis-à-vis de Celan les ouvrages suivants : Les Échos du silence, Paris : Albin Michel, 2006 [1996], p. 9, où « Corona » de Pavot et Mémoire est cité en épigraphe vis-à-vis de «l'errance», et La Pleurante des rues de Prague, op. cit., p. 47-51 et p. 128, où certaines images et idées font penser à Celan, particulièrement à La Rose de Personne.

89 Bernhard Böschenstein, dans Jean Daive, Paul Celan, les jours et les nuits, suivi de Suggestions par Werner Hamacher, trad. de l'allemand par Michèle Cohen-Halimi, Caen : Nous, 2016, p. 7-32 («Intime») (16). Böschenstein parle aussi « d'une conversion, d'une métanoïa fondamentale qui doit être amenée par la grande poésie» (13), remarque qui s'applique à celle de Tellermann. 
deuil de la brutalité exercée contre les persécutés, de l'absurde de leur disparition. La violence retenue des images devient un fil conducteur :

Un lieu soudain / fauche / lâme / s'emplit de morts et / de gibets (48)

Lettres de l'Est / s'effritent deviennent / amas de / désespoirs (67)

bouches emmêlées / de sang / carrés d'âmes / qui s'épuisent / en temple (70)

poumons d'épines / ne respirent / les lieux / les heures vides (72)

ténèbres / devinrent / flaques des enfers [...] fut fournaise de l'autre / monde (83)

Nous sommes là loin du sentiment océanique ou d'éternité que peut apporter le lyrisme. Coudre à soi les morts, faire appel à la Poésie dans l'espoir d'une entraide, voilà l'« exorcisme » dont parle la notice. ${ }^{90}$

Ces « chemins de souffle $»^{91}$ sont aussi ceux de la judéité. Née dans l'aprèsguerre, il se peut que Tellermann réfléchisse à ces chemins-là et songe à mieux les accepter. Comme l'explique Nadine Fresco, il arrivait souvent que des parents juifs transmettaient à leurs enfants la blessure de leur silence, «le vide compact» d'un «[d]euil impossible», d'une "parole impossible ».92 Tout en se méfiant de biographèmes, il ne faudrait pas écarter l'hypothèse que la crise de vers contemporaine que vit Tellermann en écrivant Le Troisième et Éternité à coudre - «une folie individuelle, intime, qui se déporte sur le collectif ${ }^{93}$ - vient en partie de là. Le côté culturel de cette folie est aussi à prendre en compte : les décennies qu'il fallut en France pour mieux intégrer les conflits des années 1940 à la conscience nationale. S'il est vrai que le deuil comporte de nombreuses étapes, il se peut que Tellermann soit en train de redécouvrir certains fils identitaires. Si elle doit retraverser la catastrophe, il s'agit tout autant

90 «Esther Tellermann : Éternité à coudre », <editionsunes.fr>.

91 Paul Celan, cité dans Renverse du souffle, op. cit., p. 187, à propos du texte définitif du Méridien et de la notion de coudre à soi les morts, de lier la poésie - au moyen du souffle - à la vie et à la mort. Cf. Paul Celan, Le Méridien \& autres proses, op. cit., p. 75-84 (82), quant aux « chemins d'une voix vers un Tu qui la perçoive».

92 Nadine Fresco, «La diaspora des cendres », L'Emprise, Nouvelle revue de psychanalyse 24 (automne 1981), p. 205-20 (208). Cf. Serge Koster, Trou de memoire : récit, Paris : PU F, 2003. « Esther Tellermann : Éternité à coudre », <editionsunes.fr >. 
de renouer, en aval, avec la tradition juive d'un savoir ouvert, en devenir, de l'ordre d'une caresse qui accueille amoureusement ce qui surgit de la parole et du livre. Tel sera souvent pour nous le sens des allusions à Celan dans Éternité à coudre, ainsi que de la figure-silhouette «Elle», la Poésie, celle qui vient à pas légers ${ }^{94}$ pour marcher à nos côtés, être anthropomorphe qui s'épanouit dès lors que nous croyons en elle, et reprise donc le possible.

«Avec toi poupée [tramer] ${ }^{95}$ : Celan peut être à la fois grave, ésotérique et gouailleur. Ce poème de Partie de neige a beau être insolent et jazzy, il n'en convoque pas moins la notion de « coudre l'éternité » à partir de l'inventivité langagière. La poésie se fait «poupée». La «carriole du chiffonnier vient jazzer », joue toujours - mais avec entrain - la même rengaine, nous vêt de vieux tissus pleins de contours et de couleurs mais sûrement démodés. Sa « trompette / bouchée / nous souffle haut dans le temps, / dans la plus dure / oreille de ce monde». Cette accusation mordante du Plus Haut et de sa parole a un certain réalisme, une certaine vérité. Selon la conception kabbaliste de l'Ein-Sof, Dieu serait le Rien mystique, sans nom et sans image, qui s'est absenté après la création mais qui reste la source de toute création. ${ }^{96}$ Le savoir reste donc hors de notre portée. Les mots et les actions nous mettent au moins sur la bonne voie. Or, la poupée-poésie, faite de vieux fils qu'il faut toujours rassembler, raccommoder, « veut avec nous / y aller », partir loin, errer, explorer, dialoguer. Il est essentiel que le lecteur lui adresse la parole, se laisse « encoign[é] » par sa musique, en saisisse le sens bienveillant ou malveillant, sache distinguer entre «Faire-du-bien» et «Faire-du-mal». Et quand cette musique s'arrête, elle reste tout de même là, une présence qui nous habite pour le meilleur ou le pire, affalée « au beau / milieu » de notre être. Celan suggère d'un air sardonique que l'homme habite toujours poétiquement, seulement il

94 Cf. Jacques Réda, Celle qui vient à pas légers, Saint Clément : Fata Morgana, 1999. Réda s'y intéresse aux cadences de la poésie plutôt qu'à l'Histoire et au mythe. Quant à Celan, il ne faut pas perdre de vue la gravité de son histoire personnelle, notamment le souvenir qu'il porte en lui d'avoir appris que sa mère avait été tuée par une balle dans la nuque, après avoir été déportée avec le père de Celan (mort par la suite «épuisé ou affamé ou victime du typhus ») dans un camp en Transnitrie. Voir par exemple Paul Celan, « Dans la lanière de priere blanche », [Renverse du souffle], Choix de poèmes, op. cit., p. 245-47 (245) : « elle [ma bouche] t’a cherchée, trace de fumée / toi, là-haut, / silhouette de femme, / toi en voyage vers mes / pensées de feu dans le gravier noir ». Les informations biographiques viennent de John E. Jackson in Paul Celan, Poèmes, op. cit., p. 23.

Paul Celan, « Mit dir Docke» / « Avec toi poupée», Partie de neige, op. cit., p. 70-71 (70; nous traduisons ces extraits). Cf. p. 198nı, à propos du caractère foncièrement " poétologique » de ce poème, notamment «le paradigme du fil» et le «dialogue hermétique » mais entraînant qui en résulte.

96 Cf. Shira Wolosky, « Paul Celan's Linguistic Mysticism », op. cit., p. 194-95. 
est impossible de faire comme autrefois. «Là-bas » reste un horizon, mais il faut rester patient et vigilant pour coudre à soi ce lieu « haut dans le temps » qui s'avère de plus en plus éloigné.

«C'est Elle / surgie de l'aiguille / non comptée » (EC 28): Tellermann réinvente ce genre d'images, suit ces mêmes chemins. Moins kabbaliste et philosophe que Celan ou d'autres, elle a néanmoins des visées similaires, à savoir explorer les «eaux cuivrées » ${ }^{97}$ de la parole, se transformer avec d'autres comme le fait Trakl - en « aiguilles aveugles » qui « grimp[ent],${ }^{98}$ suivre en nous les traces d'un « sentiment océanique »,99 atteindre par le geste poétique « les / étincelles» du langage et de l'intime qui nous relient au temps et au cosmos, mais tout autant à nos « déserts » (LT 92). Impossible de faire comme autrefois, car il y a maintenant en «Elle » - la Poésie qui nous guide et protège - les sans-nom de l'Histoire. ${ }^{100}$ Suivre ses «boucles» vers un éventuel méridien à la Celan, d'ailleurs, ce serait plus accepter de se laisser porter par l'écume et le sel du désir et, quelquefois, par les vagues de l'amertume, que de compter minutieusement des syllabes pour que le temps humain soit bien mesuré. Selon Tellermann, il s'agirait d'une parole qui « entame / la moisissure / et l'horloge / donne raison / à la voix / débauche de / rien / dans les sacs / gris » (EC 28), d'une poésie consciente des larmes de l'humanité et du temps désaccordé. Le « rien » y est à la place qui lui revient dans l'ordre cosmique, voire psychique et ontologique. C'est une «débauche de / rien », non pas du Rien : la souillure empêche toute transcendance, toute fierté. Il ne nous reste, semblerait-il, qu'à ensemencer de nouveau la parole selon cette logique de l'« entame » comme « débauche » plutôt que de donner des raisons à la voix et de suivre des chemins de souffle.

L'intéressant là-dedans, c'est l'aspect à la fois urgent et mystérieux de la présence celanienne, le glissement dans le diptyque constitué par Le Troisième et Éternité à coudre d'une relation amoureuse à une relation poétologique. « Elle », serait-ce un peu Celan lui-même, « enténébré » (LT 54) surgi de l'aiguille pour témoigner? Celan en tant qu'aiguilleur vers le désastre, vers tous

97 Osip Mandelstam, Voronezh Notebooks, op. cit., p. 50 (12-18 janv. 1937). Nous traduisons de l'anglais.

98 Georg Trakl, « Déclin », Vingt poèmes, op. cit., p. 39.

99 Sigmund Freud, Malaise dans la civilisation (1929), cité dans François-Michel Durazzo, « Guillevic et l'expérience de la limite », Guillevic : la passion du monde, op. cit., p. 85-95 (86).

100 Bien que Tellermann n'évoque pas la Shekinah, ses énoncés y font penser : soit un être féminin qui reste près de nous et accueille en elle toute l'humanité (le sens moderne proposé ici), soit un aspect féminin du divin qui reste près de nous et accueille en elle toute la lumière du cosmos (le sens proposé par la kabbale). 
les autres enténébrés ${ }^{101}$ ? Debout dans le soir, « attaché à létincelle » (LT 55), luisant ? Conscient de ceux qui l'entourent et de tous les survivants, s'adressant le cas échéant à sa femme Gisèle, à son fils Eric, à Ingeborg Bachmann, Nelly Sachs, Ilana Shmueli ${ }^{102}$ ? De manière générale, oui, cette figure qui n'apparaît qu'au passage reste perméable - mais il ne nous faut pas de réponses définitives. Retenons surtout la priorité donnée au mouvement vers d'autres êtres et le flottement temporel qu'implique celui-ci, la tension entre les temps qu'instaure la relation à l'Autre. Dans l'essai «Cristal exact», Tellermann explique que le poème celanien «étoil[ $\mathrm{e}]$ notre contingence, jusqu'à notre nom » $:$ le moi se défait, le devenir du poème nous disperse « dans un ailleurs du temps et de l'espace, une filiation identitaire mouvante », dresse « la puissance du verbe aimer $»{ }^{103}$ La vérité que nous proposa Virginia Woolf avant la Shoah, selon laquelle écrire de la poésie serait « une transaction secrète, une voix répondant à une autre voix $»,{ }^{104}$ se généralise au plan identitaire, se fait poreuse. Aussi comprend-on sans peine le fait que ce soit tantôt le Je figuré comme « aile » dans l'Autre, afin qu'ils recueillent en eux «les mourants » (LT $71 ; \mathrm{cf} .86,87)$, tantôt le Tu ou le Vous qui surgissent à l'intérieur du Je sous forme de « berges / marées d'astres / et d'âmes » (LT 94). Il faudrait retenir également que Celan, double imaginaire de Tellermann, est un être réel, qui a réellement souffert. Faire de lui un passeur de mémoire modernise le songe - nervalien ou autre et nous plonge dans un travail de mémoire engagé et volontaire.

101 À propos d'aiguilles de cendre, d'eau, de neige, de temps qui passe sur un cadran, généralement qui mettent en chemin la parole et recousent les morceaux de l'ordre symbolique éclaté, voir par exemple « Où ? » et « Toi, le », Renverse du souffle, op. cit., p. 137 et p. 173 . Cf. «Quoi coud », Partie de neige, op. cit., p. 22-25 (22; nous traduisons), à propos d'une aiguille qui fait penser aux abîmes que l'écrivain doit prendre en compte et relier à ce monde-ci : «Les abîmes / jurent par le blanc, d'où / est montée / l'aiguille de neige, / / avale-la, / / tu remets en ordre le monde».

102 Cf. Paul Celan et Gisèle Celan-Lestrange, Correspondance (1951-1970), op. cit., éd. [et trad.] Bertrand Badiou avec Eric Celan, t. I Lettres, t. II Commentaires et illustrations ; Ingeborg Bachmann et Paul Celan, Le Temps du coeur : correspondance (1948-1967), augmentée des lettres échangées par Paul Celan et Max Frisch ainsi que par Ingeborg Bachmann et Gisèle Celan-Lestrange, éd. Bertrand Badiou, Hans Höller, Andrea Stoll et Barbara Wiedemann, trad. Bertrand Badiou, Paris : Seuil, 2011 ; Nelly Sachs et Paul Celan, Correspondance, trad. Mireille Gansel, Paris : Belin, 1999 ; Paul Celan et Ilana Shmueli, Correspondance (19651970), éd. Ilana Shmueli et Thomas Sparr, trad., révision et adaptation des notes Bertrand Badiou, Paris : Seuil, 2006. Souvent, les lettres et les poèmes de Celan s'intriquent. Il n'est pas rare qu'il écrive un poème pour un destinataire précis. Généralement, le traducteur de la correspondance explique les circonstances qui ont mené à l'écriture du texte poétique. 
Le drame éclate lorsque ce travail s'interrompt et que la contingence s'embrume. L'inconscient résiste au travail de mémoire, pour on ne sait quelle raison. Petit à petit, la nuit - au sens propre comme au figuré - est franchie et porte conseil. Quelque chose de revigorant arrive, au fur et à mesure que la parole s'éploie et que le moi redevient poreux. Explorons maintenant comment Tellermann parvient à « porter » jusqu'à soi l'Autre (LT 97) pour mieux appeler « ceux d'en bas », cueillir «leur soif » et leur donner accès à la contingence, au possible que nous ouvre la parole, au « Troisième / et [au] songe » (LT 99). Concentrons-nous sur le tissu allusif et la manière dont ces drames intérieurs se résolvent. Les premiers mots, par exemple, renouent avec l'énigme en acceptant l'étroit. La trajectoire sera celle de la prise en compte du souffle poétique en tant qu'il appartient à tous, les morts et les vivants, mais ne s'ouvre que si l'on accepte ses silences et parvient à y frayer à plusieurs un passage. Les vers « Nuque / à peine» $(E C 7)$ annoncent cette problématique. Normalement, la nuque serait un lieu essentiel au bien-être corporel, un centre du système nerveux, pulmonaire et alimentaire, ainsi qu'un de nos liens au sacré grâce aux gestes de dévouement qu'elle nous permet. C'est comme s'il fallait quitter le temps et l'espace quotidiens pour se situer dans un autre espace-temps identitaire, celui où l'on se pétrit « de mots et / d'absence », de « rien », de toute « première parole » par laquelle on se fraie des sentiers identitaires malgré « ce trop / dans la bouche » (7-8). Tâche difficile, inscrite dans le dialogue avec un Tu et faisant écho à toute une série de poèmes dans Renverse du souffle, où, de même, on se trouve avec un Toi blessé, veillant par la voix auprès de l'Autre « en plein milieu de la nuit », « entouré de pauses ». ${ }^{105}$ Travail charnel et psychique, l'affaire des mains en train de pétrir, celles de l'être humain qui cherche par sa voix et aussi sans voix « un chemin ». 106

La descente en soi n'est pas facile. Cela aide de suivre les sentiers qu'ouvre le dire celanien. Il faut se plonger dans le rite par lequel Tellermann accepte « un face à face », arrache à l'Autre « la lenteur / un paysage ou encore / une syllabe que / le temps ponctue» (9). Ces vers se réfèrent à une lente marche à deux au moyen des signes, ainsi qu'à la « douleur» qui sous-tend le défi de nommer le «sans mort», bégayer le désarroi, l'«é-, é-, é- / pel[er] ${ }^{107} \mathrm{Lu}$ à

105 Paul Celan, « Schieferäugige » / « Celle aux yeux d'ardoise » et « Ruh aus in deinen Wunden » / « Repose-toi dans tes blessures », Renverse du souffle, op. cit., p. 168-69 et p. 178-79 (168 et 178 ; nous traduisons). Cf. toute la cinquième partie de Renverse du souffle, ainsi que « Playtime », Partie de neige, op. cit., p. 103.

106 Paul Celan, «Lettre à Hans Bender », 8 mai 196o, Le Méridien \& autres proses, op. cit., p. $43-45(44)$.

107 Paul Celan, « Die Silbe Schmerz » / « Les syllabes douleur », La Rose de Personne, op. cit., p. 130-33 (130, 132; nous traduisons). En allemand, le mot «douleur» (Schmerz) n'a qu'une syllabe. 
travers des vers d'un autre recueil de Tellermann, ce passage d'Éternité à coudre suggère bien le temps qu'il faut pour naviguer à plusieurs sur la « soie » de la parole et « l'amertume » (CE 205) de l'Histoire. Tellermann doit transposer en quelque sorte l'allemand de Celan aux moments où elle songe à une certaine tonalité, par exemple pour reproduire la même qualité saccadée et consonantique. C'est peut-être en ce sens qu'il faut lire l'éventail d'images dans Éternité à coudre: celui des fils qui modulent le dire par leurs poignées de syllabes, des divers mots-cailloux à partir desquels étincellent des embruns sémantiques. De même, on prend conscience de la fluidité symbolique du langage, par exemple là où Tellermann décrit son « double » celanien par les vers «tu greffes au ciel / la syllabe / elle / neige / et fleurit » (EC 69). Le langage s'intègre ainsi aux rythmes du cosmos, au cycle des saisons, au temps qui passe. Le livrepoème y est « pore » infime qui reçoit les dons des mondes intérieur et extérieur, afin de les faire éclore « comme un autre / soleil » (53). Même les alinéas et espacements sont rythmés, mesurés - $3,5,7$-, ce qui confère aux poèmes un caractère sacré108 et «ce vibrato douloureusement muet »109 que l'on ressent également dans Le Troisième.

Surgit de cette étoffe, des «océans » et « rumeurs » (11), une sorte d'image rémanente : «Elle», la Poésie, non pas celle qui viendrait par de beaux mots sur la page, mais une sorte d'espace intérieur personnifié, suite au geste sacré par excellence d'avoir brûlé sous le nom de l'Autre « des cendres » (12). Un Tu « gliss[e]» à travers la peau et « occup[e]» les espaces «d'Elle» (15). C'est comme si, en «défaisant» ce qu'elle savait des «deuils» (12), Tellermann laissait travailler son inconscient, voyait quelque part dans son âme cet Autre - pour nous, il s'agirait de Celan -, et imaginait dans ces profondeurs un témoin-passeur, un Autre dont s'occuper pour que s'effectue un transfert affectif qui permette à la parole de se renouveler. Elle lui lave la « bouche», épuise

108 Voir aussi la note 64 ci-dessus à propos du chiffre trois et de l'ordre parfait du cosmos. Quant au chiffre sept, aussi présent que le trois dans les énoncés tellermanniens - « Où est / la maison / nos 3 / solitudes ? » (EC 26); « cendres de 3 / souffles » (EC 45); « Tu viens je / me dépouille / des 7 noms» (LT 73); «Sept couleurs / pourraient / réchauffer / le dedans / et les aubes » (EC 82) -, voir Jean Chevalier et Alain Gheerbrant, éd., Dictionnaire des symboles, op. cit., p. 86o-61, et Nanon Gardin et al., Petit Larousse des symboles, op. cit., p. 570-72: «Sept correspond [...] aux sept degrés de la perfection, aux sept sphères ou degrès célestes [...] Il était chez les Égyptiens symbole de vie éternelle. [...] [C'est] le symbole [...] d'une totalité en mouvement ou d'un dynamisme total »; « Les douleurs de la Vierge [...] sont à l'origine du culte de Notre-Dame-des-Sept-Douleurs ». Pour ce qui est du cinq, voir Gardin, p. 162-63: « Nombre des doigts de la main et des sens, le cinq est le chiffre de l'homme, de l'harmonie et de l'équilibre », symbole « de l'alliance et de la protection », « de la perfection », « de la grâce divine ».

Paul Celan, « La poésie d'Ossip Mandelstam », op. cit., p. 11. 
sa propre « ténèbre », « bouffées d'alphabets / et de palmes » (15), comme pour dire que témoigner serait l'affaire de chaque génération de poètes: dans la symbolique celanienne, ces palmes seraient l'indice de mondes premiers que sème le dire poétique. ${ }^{110}$ Le syntagme «bouffées d'alphabets» (15), renvoie d'ailleurs aux vers évoquant l'idée de « suivre / le nerf / jusqu'à la mémoire / où poussent / de vieux alphabets » (54), phrase qui peut faire penser aux textes sacrés du monde entier.

En effet, pour que ces rites purificateurs soient réussis, il faut s'allier à autrui à travers «Elle», la Poésie. «Elle» est reléguée au second plan, sauf dans les vers « c'est elle / talons sur les / planches / c'est Elle / épuisant / le cœur / sa verdure / devra devra / venir » (29) et « Elle / épuisant le cœur / lumière à cru / avance monte / ruine / à bas bruit / les interstices [...] voulut / dépouiller de toi / les buissons » (36). Or, lu au travers d'une allusion à Celan, il ne s'agit pas que d'images universelles ayant trait à la poésie et au renouveau, mais des pas d'« Elle », celle qui « racle » le dire afin de parler et d'écrire avec justesse, éclat, précision, véracité, surtout en veillant à respecter « les poursuivis ».111 Nous avons évoqué plus haut la «caresse » du langage par laquelle on s'occupe du savoir et de notre devenir commun. ${ }^{112}$ Le fascinant ici est de voir une mise en scène de ce principe. Le Je lyrique ne sonde pas que les profondeurs de sa propre âme, mais, aux côtés d'«Elle», celles de tous ceux qui ont souffert de l'exil et de la différence. Si « folie »113 il y a, cette générosité

110 À propos de «fougères palmées » qui en s'élèvant vers le ciel connotent ce qui métaphoriquement peut y éclore, briller, emplir l'espace de frondes à la fois primitives et annonciatrices, et transformer ainsi la sensation et la perception, cf. Paul Celan, «Pierre de vue aciérugineuse », Partie de neige, op. cit., p. 123 et p. 233n3.

111 Paul Celan, «Mit den Verfolgten » / «Avec les persécutés », Renverse du souffle, op. cit., p. $3^{6-37}$ ( 36 ; nous traduisons), qui se réfère à un pas en quelque sorte très humain, en l'occurrence au «talon » qui écrit et qui s'achemine ainsi avec les poursuivis, ceux qui sont traqués ou exilés et que le langage doit réintégrer à l'ordre social et symbolique, voire divin et ontologique. Voir aussi p. 206n5, à propos des sefirot qui «montrent l'abîme divin» tout en ne laissant voir «que leurs talons ». Selon Geoffrey Wigoder (sld), Dictionnaire encyclopédique du judaïsme, Paris : Cerf/Robert Laffont, 2012, p. 938, les sefirot sont les « dix émanations à travers lesquelles se manifeste la Divinité » et qui forment «l'unité dynamique » de l'arbre de vie si cher à la mystique juive. Voir plus haut à propos de l'Ein-Sof, autre nom pour la Divinité elle-même.

112 Marc-Alain Ouaknin, Lire aux éclats : éloge de la caresse, op. cit., p. 38 et p. 61. Voir aussi Sylvie Germain, Le Vent ne peut être mis en cage, l'intégrale des entretiens Noms de Dieux d'Edmond Blattchen, Bruxelles : Alice Éditions et RTBF Liège, 2002, p. 21-23, à propos d'une attitude à l'égard de Dieu qui serait de l'ordre de la caresse et qui aurait à voir avec l'échange, la reconnaissance, la tolérance, la patience, tout ce que l'on construit intérieurement.

« Esther Tellermann : Éternité à coudre », <editionsunes.fr >. 
intersubjective la rend finalement bénéfique. «Elle », la poésie consciente de la catastrophe, facilite la traversée de paysages noircis, le travail de mémoire sur ce que l'on sait sans le savoir vraiment, sur le « gris » qui « happe la profondeur / d'elle » (57) et pèse sur l'inconscient. L'auteur « traverse l'étroitesse »114 de son souffle tout en discernant des figures, réelles ou imaginaires, qui font s'étendre celui-ci, montrent des voies et des gestes vers d'autres seuils.

Tellermann poursuit le développement et le dénouement de ce récit d'un air patient qui comprend l'obligation de regarder de près l'horreur. Elle s'en sort indemne puisqu'elle est si peu seule. Le fait de regarder avec autrui à travers une grille de parole - « tant voulions herses » (26) - et d'entendre dans le rêve des voix l'apaise. Même si elle doit passer par la douleur, plusieurs énoncés suggèrent que son trouble va prendre fin. Par exemple, les mots sont des fils métaphoriques de diverses sortes qui l'aideront à coudre l'éternité : « notre auge », «notre abri », «des meules », « des sentiers sevrés » (40). Il ne s'agit là nullement de plénitude, mais plutôt de retrouvailles avec soi, autrui et le monde au moyen de la parole. La page suivante dépeint une scène non loin des tableaux du peintre russe Marc Chagall (1887-1985), en plus existentielle et féérique: «Si lentement / me pénètre / votre fil / nos mains rescapées / dessinent / des dehors / effleurent votre / bouche » (41). C'est un poème poétologique sur des voix qui s'entrecroisent, mais où ces «mains rescapées » semblent laisser l'empreinte sur la page écrite de corps revenus de leur exil au premier plan et de vastes paysages au fond, plans où d'ailleurs les plans euxmêmes s'entrecroisent lorsque « parmi / vous grimpe / je ne sais / l'églantier » (41), image qui recèle un à-venir de la parole, d'éventuels rosiers en dépit des épines métaphoriques, tel le chant des « roseaux » qui continuera peut-être à plier derrière le monde « les amertumes » (42). Particulièrement importants, étant donné «l'orage » annoncé peu après lorsque « soir [...] s'incline » (43), sont les vers «à présent je ne / crains les dedans / qui déracinent » (41), car s'ensuivent par-ci par-là des images évoquant la catastrophe et le devoir-vivre juif. ${ }^{115}$ En contraste avec la supplication désespérée qui clôt Le Troisième, «Te supplie / encore appelle / ceux d'en bas » (LT 99), s'insère dans Éternité à

114 Ibid. : « la langue comme existence traverse l'étroitesse de celui qui écrit le poème ».

115 Nous empruntons à Jean-Pierre Lefebvre l'idée du devoir-vivre, expression employée dans ses commentaires. Cf. Paul Celan, « Tu peux », Renverse du souffle, op. cit., p. 9, et p. 190-93. Comme nous le rappelle Lefebvre, chaque poème qu'écrit Celan l'aide à traverser des périodes très difficiles - hantées par la mort, dont il faut se servir lorsqu'on crie sa douleur et accueille la neige de la parole - et nous encourage à en faire de même. Une telle traversée apaisera l'âme et fera reverdir le champ poétique, en y inscrivant le relationnel et « la brûlure du devoir-vivre » (193). 
coudre une certaine cambrure de soi et du monde : « une nuque et / l'écorce » $\left(E C 5^{2}\right)$ prêtes à chanter; le « concert de talons / et de voix » (55) que le lecteur doit entendre dans l'« espace » (46) intérieur d'autrui ; les « fronts sanglés » en prière qui « se / retournent et / détressent / l'écho » (46).116

Après avoir fait face dans Le Troisième et Éternité à coudre à une période difficile, l'auteur s'affranchit d'un fardeau psychique. Son deuil pour un pair-témoin s'achève - « c'est bien maintenant » (69) - sans fin définitive. « Le Troisième », syntagme qui correspond chez Tellermann à l'espace-temps de l'union symbolique, a pu luire. « Là pic » a permis aux « éternités »- aux profondeurs affectives, linguistiques, ontologiques, historiques - de se laisser «coudre » $(E C \quad 26)$ dans et par la parole. La trajectoire de seuil en seuil peut continuer. L'« erre » désemparée reste dans le passé, « ensevelit » (86) des bien-aimé(e)s, même si l'on continue à héler le navire du deuil et son passeur, témoigner pour d'autres, se coucher « entre » eux (91). ${ }^{117}$ Le dire se calme. Des « souvenirs [...] se rapatrient $\gg(73)$.

En regardant disparaître au loin l'embrun des syllabes, en prêtant l'oreille à leurs échos, songeons au vibrato venu de l'Est et à la pensée syncrétique de Tellermann. Lorsque l'auteur traverse des aires littéraires, elle nous fait percevoir d'importants pans d'idées religieuses et philosophiques, par exemple là où l'acheminement et le non-savoir sont hautement valorisés. Sont parfois suggérées à cet égard des traditions judaïques : l'amour pour la «lettre » qui nous « ouvre » $(75 ; \mathrm{cf} .43,81)$ à l'ombre et à la lumière, le mysticisme linguistique par

116 Cf. Paul Celan, «Dans la lanière de prière blanche », Renverse du souffle, op. cit., p. 71, et Choix de poèmes, op. cit., p. $365 \mathrm{n} 1$ : «Il s'agit des tefillin [...], deux petites boîtes de cuir contenant quatre passages de la Bible que les hommes portent pour la prière du matin en semaine, au bras gauche et sur la tête. [...] Ces boîtes sont tenues par des lanières. » Ainsi ces « fronts sanglés » (EC 46), à lier par exemple dans l'imaginaire collectif aux hommes juifs très pieux de « Jérusalem » (43) ou de « l'Est » (67).

117 Cf. « Couche ta tête entre / toi » $(E C 53,55)$; « j'ai voulu [...] me coucher entre / vous » (TE $221)$; « je me suis couchée entre / toi » (TE 258); «J'avais couché entre / toi » (SN 246). Cf. Paul Celan, «Strette», [Grille de parole], Choix de poèmes, op. cit., p. 157, «C'est moi, moi, / moi qui étais couché entre vous », où « vous » se réfère aux disparus, autant de la Shoah que de la bombe atomique. Voir aussi «Le tambour de bateleur», [Renverse du souffle], Choix de poèmes, p. 257, le vers « je suis couché près de toi », ainsi que le poème « Largo », Partie de neige, op. cit., p. 51. Comme c'est le cas pour EC 53 et 55, il est possible de se figurer aussi un seul être qui se sent divisé. Une telle crise existentielle se présente dans le récit de Celan, Entretien dans la montagne, trad. John E. Jackson et André Du Bouchet, Saint Clément : Fata Morgana, 2017. Dans une lettre à Adorno à l'égard de ce récit, Celan emploie les mots « le Troisième » pour désigner celui qui se tient à distance de son dilemme psychique, de sa division, et parvient à se consoler en témoignant. Voir Andréa Lauterwein, Paul Celan, op. cit., p. 163-66. 
lequel nous tâchons de mieux habiter la parole, la passion que l'on peut avoir pour des figures protectrices. ${ }^{118}$ Toujours très en évidence : «le battement » (78) du texte, ses archipels, «la jouissance de la lettre» et « du mot».119 L'intersubjectivité, enfin, ne cesse d'infléchir la parole tellermannienne. Ces livres traitent du désir de suivre ses semblables, de porter en soi au niveau du souffle la « rumeur » d'autrui pour mieux la « disperser » $(54 ; \mathrm{cf} .11,13)$. Si par moments dans Éternité à coudre la locutrice ou la Poésie se dédoublent, cela nous rappelle combien l'amour et les signes qui les sous-tendent font partie d'un « désir inépuisable », 20 tissent un « vêtement ${ }^{121}$ qui nous relie, de recueil en recueil, au monde et à soi.

118 Nous pensons à la Shekinah dans la tradition kabbaliste et au Golem. La figure-silhouette protectrice qui apparaît dans Éternité à coudre a quelques liens avec ce dernier, dans la mesure où son existence dépend du langage et de notre croyance en elle, nous habite mais peut-être de façon éphémère. Cf. les propos d'Anne Teyssiéras cités dans John C. Stout, «Anne Teyssiéras », L'Énigme-poésie : entretiens avec 21 poètes françaises, Amsterdam : Rodopi, 2010, p. 81-97 (93-94).

119 Sophie Nauleau, « Entretien avec Esther Tellermann », op. cit.

120 Sylvie Germain, Ateliers de lumière: Piero della Francesca, Johannes Vermeer, Georges de La Tour, Paris : Desclée de Brouwer, 2004, p. 56 : «L'étonnement, qui ouvre le cour et la conscience des femmes à une quête silencieuse, lance l'esprit des hommes dans l'aventure d'une conquête. Chez les femmes, l'étonnement se fait amour et l'amour désir inépuisable.»

121 Luce Irigaray, Être deux, op. cit., p. 87: «Le désir d'aimer reste encore et toujours un vêtement tissé, aussi bien par moi que par toi, de terre et de ciel, de nuit et de lumière, d'ombre et de soleil. Et ainsi à l'infini. » 